\title{
TITLE:
}

\section{High energy neutrino early afterglows from gamma-ray bursts revisited}

\author{
$\operatorname{AUTHOR}(S)$ :
}

Murase, $\mathrm{K}$

\section{CITATION:}

Murase, K. High energy neutrino early afterglows from gamma-ray bursts revisited. PHYSICAL REVIEW D 2007, 76(12): 123001.

ISSUE DATE:

2007-12

URL:

http://hdl.handle.net/2433/50480

RIGHT:

Copyright 2007 American Physical Society 
PHYSICAL REVIEW D 76, 123001 (2007)

\title{
High energy neutrino early afterglows from gamma-ray bursts revisited
}

\author{
Kohta Murase \\ Yukawa Institute for Theoretical Physics, Kyoto University, Oiwake-cho, Kitashirakawa, Sakyo-ku, Kyoto, 606-8502, Japan \\ (Received 8 July 2007; published 4 December 2007)
}

\begin{abstract}
The high energy neutrino emission from gamma-ray bursts (GRBs) has been expected in various scenarios. In this paper, we study the neutrino emission from early afterglows of GRBs, especially under the reverse-forward shock model and late prompt emission model. In the former model, the early afterglow emission occurs due to dissipation made by an external shock with the circumburst medium (CBM). In the latter model, internal dissipation such as internal shocks produces the shallow decay emission in early afterglows. We also discuss implications of recent Swift observations for neutrino signals in detail. Future neutrino detectors such as IceCube may detect neutrino signals from early afterglows, especially under the late prompt emission model, while the detection would be difficult under the reverseforward shock model. Contribution to the neutrino background from the early afterglow emission may be at most comparable to that from the prompt emission unless the outflow making the early afterglow emission loads more nonthermal protons, and it may be important in the very high energies. Neutrino detections are inviting because they could provide us with not only information on baryon acceleration but also one of the clues to the model of early afterglows. Finally, we compare various predictions for the neutrino background from GRBs, which are testable by future neutrino observations.
\end{abstract}

DOI: 10.1103/PhysRevD.76.123001

\section{INTRODUCTION}

Gamma-ray burst (GRB) is one of the most energetic phenomena in the Universe and one of the candidates where both electrons and protons are accelerated up to very high energies. If protons are accelerated up to very high energies, we can expect high energy neutrinos and gamma rays that are produced by the photomeson production process. We investigated the high energy neutrino emission from GRBs under the internal shock model in the previous work [1]. Since the prediction by Waxman and Bahcall [2], this kind of neutrino emission has been discussed by several authors (see, e.g., [3-5]).

The standard (internal-external) shock model of GRBs succeeded in explaining many observations in the pre-Swift era (see reviews, e.g., [6-8]). Synchrotron radiation from a reverse shock (RS) and a forward shock (FS) usually peaks in the infrared-to-optical or even lower bands and ultraviolet-to-x ray bands, respectively. Some of the infrared/optical flashes that can be interpreted as the RS emission were observed from GRB 990123 [9-18], GRB 021211 [19-21], GRB 021004 [22,23], and GRB 041219a [24-26]. These infrared/optical photons can interact with protons accelerated at the RS and generate high energy neutrinos. Under the RS model, Waxman and Bahcall predicted high energy neutrino afterglows for the homogeneous circumburst medium (CBM) (or interstellar medium (ISM)) [27]. For the windlike CBM, Dai and Lu predicted $\left(10^{15}-10^{17}\right)$ eV neutrinos [28]. Dermer [29,30] and Li et al. [31] considered the neutrino emission from the FS, assuming that protons can be accelerated via the second-order Fermi acceleration mechanism. Now, large

\footnotetext{
*kmurase@yukawa.kyoto-u.ac.jp
}

PACS numbers: 95.85.Ry, 14.60.Lm, 25.20.-x, 98.70.Rz

neutrino detectors such as IceCube [32] and KM3Net [33] are being constructed. By exploiting these detectors, we can test various predictions for high energy neutrinos from GRBs in the near future. Furthermore, ANITA [34] and Auger [35] might also be useful for detecting very high energy neutrinos.

Such possibilities for the neutrino emission have been studied under the standard model. However, the recent observations by the Swift satellite have shown many unexpected behaviors in the early afterglow phase and the simplest standard model is confronted with difficulties (see e.g., [36-39]). For example, one of them is the shallow decaying behavior, which appears after the steep decay phase. The flux decay becomes shallow, when the flux decays as $\propto t^{-(0.2-0.8)}$ up to a break time $\sim 10^{3-4} \mathrm{~s}$ $[40,41]$. Although various modifications of the standard model have been put forward to explain the observations, none of which seems conclusive.

In addition, the central engine may last much longer than the duration of the bursts. The variability of some GRB afterglows implies such a prolonged activity of the central engine [42]. Flares that are found in a significant fraction of Swift GRBs require a long duration and/or reactivation of the central engine, and one of the leading models for explaining flares is the late internal dissipation model $[43,44]$. Furthermore, such late internal dissipation may explain the shallow decaying behavior [45]. After the earlier prompt emission, there may be a tail of activity of the central engine, producing subshells of progressively lower power and bulk Lorentz factor for a long time.

The rapid follow-up by the Swift satellite also allows us to observe many early afterglows of GRBs in the optical bands by ground-based robotic telescopes such ROTSE. However, many ground-based and Swift UVOT observa- 
tions have shown that the majority of bursts have very dim or undetectable optical afterglows [46]. Although foreground extinction, circumburst absorption, and high redshift provide explanations for many GRBs, there is tentative evidence that the strong RS emission is suppressed. Theoretically, there are some possible reasons for this paucity of previously predicted optically bright flashes, if they are ascribed to RS emission. One is the absence or weakness of the RS. It is because earlier simplified estimates can overpredict the optical emission when the RS is semirelativistic $[47,48]$ and/or the optical emission can be much weaker when the ejecta is highly magnetized [49]. Alternatively, Beloborodov pointed out the RS emission can be suppressed enough if the prompt emission overlaps with the RS emission [50]. On the other hand, some authors suggested that the RS emission is seen as the shallow decay emission [51,52]. They argued that significant energy of such the RS emission may be radiated in $\mathrm{x}$-ray bands and the shallow decay phase can be explained by the RS running into the ejecta of relatively small Lorentz factors.

Although many models have been proposed, there are no conclusive ones for explaining early afterglows. More and more observations will be needed in order to discriminate them. High energy neutrino and gamma-ray observations can provide us one of the useful clues to such models. In this paper, we focus mainly on the high energy neutrino early afterglow emission. We calculate neutrino spectra more quantitatively than previous works under the various early afterglow models. The method of calculation is the same as that used in our previous works [53,54], where we use Geant4 [55] with experimental data [56,57]. Future observations of high energy neutrinos could provide us with not only information on baryon acceleration but also physical conditions of GRBs. Combined with observations of electromagnetic emissions, they would be useful as one of the clues to the early afterglow models, although neutrino telescopes that are larger than IceCube will be desirable.

This paper is structured as follows. In Sec. II A, we briefly review the reverse-forward shock model. Then, we consider contributions from the early and late prompt emission in Secs. II B and II C. In Sec. II, we review the high energy neutrino emission process in GRBs. The numerical results are shown in Sec. IV. We describe summary and discussions in Sec. V.

\section{THE MODEL}

\section{A. The reverse-forward shock model}

The original reverse-forward shock model, which is successful in interpreting many late time afterglow observations basically, has been developed (see reviews, e.g., $[6-8,12,39])$ and often accepted on interpreting some early infrared/optical flashes such as GRB 990123. Here, we briefly review this model which is used for our calculations
$[17,21]$. The analysis in this subsection is entirely within the context of the external shock model. We use this model only for the afterglow emission, and assume that the prompt emission occurs due to internal dissipation such as internal shocks. The possibility that internal dissipation makes the early afterglow emission is described in Sec. II C.

The expanding fireball strikes the surrounding medium and will form two shocks: a reverse shock and a forward shock. The shocked ambient and ejecta materials are in pressure balance and are separated by a contact discontinuity. In the original standard model, the RS is thought to be short lived, which exists during the initial deceleration of the fireball. After the RS crosses the ejecta, the FS continues and the ejecta will transit to self-similar expansion which is described by the Blandford-Mckee solution.

Each shock compresses the fluid. Let the Lorentz factor of the shocked CBM and that of the shocked ejecta (measured in the frame of the unshocked CBM) be labeled by $\Gamma$. $\Gamma^{\prime}$ is the Lorentz factor of the unshocked ejecta (measured in the frame of the shocked ejecta), which is written as

$$
\Gamma^{\prime} \approx \frac{1}{2}\left(\frac{\Gamma}{\Gamma_{0}}+\frac{\Gamma_{0}}{\Gamma}\right),
$$

where $\Gamma_{0}$ is the Lorentz factor of the unshocked ejecta (measured in the frame of the unshocked CBM) and we have assumed $\Gamma_{0}, \Gamma \gg 1$. By combining this equation with the pressure equality at the contact discontinuity, we have

$$
\Gamma \approx \frac{\Gamma_{0}}{\left[1+2 \Gamma_{0}\left(n / n_{\mathrm{ej}}\right)^{1 / 2}\right]^{1 / 2}} .
$$

Here the number density of the ejecta, $n_{\mathrm{ej}}$, is given by $n_{\mathrm{ej}}=$ $E_{\mathrm{ej}} / 4 \pi m_{p} c^{2} \Gamma_{0}\left(\Gamma_{0} \Delta\right) r^{2}$, where $E_{\mathrm{ej}}$ is the isotropic energy of the ejecta and $\Delta$ is the geometrical thickness of the ejecta measured in the stellar frame. The circumburst number density is written as $n=A r^{-k}$, where $k=0$ expresses a homogeneous CBM (ISM) and $k=2$ does a windlike CBM. In the latter case, $A=3 \times 10^{35} A_{*} \mathrm{~cm}^{-1}$, where $A_{*}$ is the mass-loss rate to wind speed ratio, normalized to $10^{-5} M_{\odot} \mathrm{yr}^{-1}$. $A_{*} \sim 1$ is a typical value for WalfRayet stars [58].

Now, we define the crossing radius $r_{\times}$, at which the RS finishes crossing the ejecta and injection of fresh electrons by the RS ceases. The thickness of the ejecta at the crossing time $t_{\times}$is written as $\Delta\left(r_{\times}\right)=\int_{0}^{r_{\times}} d r\left(\beta_{0}-\beta_{\mathrm{RS}}\right)$, where $\beta_{0}-\beta_{\mathrm{RS}}=1.4 \Gamma_{0}^{2} n^{1 / 2} / \Gamma_{0}^{2} n_{\mathrm{ej}}^{1 / 2}$ is the difference between the speed of the unshocked ejecta in the stellar frame and that of the RS. This expression is valid for a wide range of the ratio $\Gamma_{0}^{2} n / n_{\mathrm{ej}}$, from Newtonian to relativistic RS cases $[17,21]$. If the ejecta thickness is smaller than the spreading radius which is given by $r_{\mathrm{s}} \approx 2 \Gamma_{0}^{2} c T$, the ejecta thickness at the crossing time $\Delta_{\times}$is expressed as $\Delta_{\times} \approx \Delta_{0} \equiv c T$. This case is called a thick ejecta case in the usual terminology [59]. Here, $T$ is the duration of the GRB ejecta in 
the stellar frame. If the ejecta thickness is larger than $r_{\mathrm{s}} / 2 \Gamma_{0}^{2}$, we have $\Delta_{\times} \approx r / 2 \Gamma_{0}^{2}$. This case is called a thin ejecta case. We can write the crossing radius in the thick ejecta regime as follows:

$$
r_{\times}=\left\{\begin{array}{lll}
0.24 \times 10^{17}\left(\frac{E_{\mathrm{ej}, 53} T}{n}\right)^{1 / 4} \mathrm{~cm} & (k=0) \\
0.52 \times 10^{15}\left(\frac{E_{\mathrm{ej}, 53} T}{A_{*}}\right)^{1 / 2} \mathrm{~cm} & (k=2)
\end{array} .\right.
$$

In the thin ejecta regime, we have the following expressions:

$$
r_{\times}=\left\{\begin{array}{ll}
0.44 \times 10^{17}\left(\frac{E_{\mathrm{e}, 53}}{n \Gamma_{0,2.5}^{2}}\right)^{1 / 3} \mathrm{~cm} & (k=0) \\
1.0 \times 10^{14}\left(\frac{E_{\mathrm{e}, 53}}{A_{*} \Gamma_{0,2.5}^{2}}\right) \mathrm{cm} & (k=2)
\end{array} .\right.
$$

The essential length scales for the thick and thin ejecta cases can be expressed as $r_{\Delta} \equiv l^{3 / 4} \Delta_{0}^{1 / 4}$ and $r_{\Gamma} \equiv l \Gamma_{0}^{-2 / 3}$, respectively. Here, $l$ is the Sedov length. For $k=0$, we have $l \equiv\left(E_{\mathrm{ej}} /(4 \pi / 3) n m_{p} c^{2}\right)^{1 / 3}$. Using these length scales, we can write the crossing radius $r_{\times} \sim$ $\max \left(r_{\Delta}, r_{\Gamma}\right)$. It is often convenient to introduce $\xi \equiv$ $\left(r_{\Gamma} / r_{\Delta}\right)^{2}=\left(l / \Delta_{0}\right)^{1 / 2} \Gamma^{-4 / 3}$. Note that, if $\xi \gtrsim 1$, the ejecta is essentially in the thin ejecta regime. If $\xi \lesssim 1$, it is essentially in the thick ejecta regime.

The crossing time is calculated by $t_{\times}=$ $\int_{0}^{r \times} d r\left(1 / 2 \Gamma^{2} c\right)$. For a thick ejecta case with $k=0$, we can obtain $t_{\times} \approx 17 \mathrm{~s}\left(\frac{E_{\mathrm{e}, 53}}{n \Gamma_{0,2.5}^{8}}\right)^{1 / 3}$. For a thick ejecta case with $k=2, t_{\times} \approx 2.9 \mathrm{~s}\left(\frac{E_{\mathrm{e}, 53}}{A_{*} \Gamma_{0,2}^{4}}\right)$. For thin ejecta cases with $k=0$ and $k=2$, we have $t_{\times} \approx 0.72 \mathrm{~s} \times T$. Up to an order-unity factor, the crossing time can be approximated as $t_{\times} \sim$ $\max \left[T, t_{\Gamma}\right]$. Here, the crossing time is regarded as the time when afterglows begin, i.e., the self-similar behavior starts. The RS emission rises after the ejecta starts to sweep up matter, but it does not peak until time $\sim t_{\times}$, marking the beginning of the self-similar regime. Most of the RS emission (hence the neutrino emission from the RS) is released at $\sim t_{\times}$. Therefore, we can expect that it is sufficient to consider the behavior at $t_{\times}$for our purpose of evaluating the neutrino energy fluence, although more sophisticated calculations including the time dependent evolution are possible [60].

The magnetic fields in each post-shock fluid are parametrized by fractions $\epsilon_{B}^{f}$ and $\epsilon_{B}^{r}$ of the post-shock internal energy density stored in it, respectively. They are written as

$$
\begin{aligned}
& B_{\times}^{f}=\sqrt{32 \pi \epsilon_{B}^{f} n m_{p} c^{2}\left(\Gamma_{\times}-1\right)\left(\Gamma_{\times}+3 / 4\right),} \\
& B_{\times}^{r}=\sqrt{32 \pi \epsilon_{B}^{r} n_{\mathrm{ej}} m_{p} c^{2}\left(\Gamma_{\times}^{\prime}-1\right)\left(\Gamma_{\times}^{\prime}+3 / 4\right) .}
\end{aligned}
$$

The injection Lorentz factor of electrons at the crossing time is

$$
\gamma_{e, m}=\frac{\epsilon_{e}}{f_{e}} g(p) \frac{m_{p}}{m_{e}}\left(\Gamma_{\times}^{(\prime)}-1\right),
$$

where $g(p)$ is given by $g(p)=(p-2) /(p-1)$ and $f_{e}$ is a number fraction of the shocked electrons that are injected into the acceleration process. The cooling Lorentz factor of electrons is obtained by equating the cooling time and dynamical time $t_{\mathrm{dyn}}=r_{\times} / \Gamma_{\times} c$, which is written as

$$
\gamma_{e, c}=\frac{6 \pi m_{e} c^{2} \Gamma_{\times}}{\sigma_{\mathrm{T}} B_{\times}^{2} r_{\times}(1+Y)},
$$

where $Y$ is the Compton parameter, which can be calculated from the electron distribution (see e.g., [61]).

The injection energy $\varepsilon^{m}=h \nu_{m}$ and cooling energy $\varepsilon^{c}=h \nu_{c}$ in the comoving frame are calculated from the corresponding electron Lorentz factors by using $\varepsilon=h \nu \approx$ $\hbar \gamma_{e}^{2} \frac{e B_{\times}}{m_{e} c}$. In this paper, we consider the four cases; the thick ejecta colliding into the ISM, the thin ejecta colliding into the ISM, the thick ejecta colliding into the windlike CBM, and the thin ejecta colliding into the windlike CBM. For example, let us consider the case of the thin ejecta colliding into the ISM. We can obtain two characteristic frequencies in the observer frame $\left(\nu^{\mathrm{ob}} \simeq \Gamma_{\times} \nu\right)$ as follows. For the FS emission, we have $\nu_{m, f}^{\mathrm{ob}} \approx 2.9 \times 10^{20} \mathrm{Hzg}^{2}\left(\epsilon_{B,-2}^{f}\right)^{1 / 2} \times$ $\left(\epsilon_{e,-1}^{f}\right)^{2}\left(f_{e}^{f}\right)^{-2} E_{\mathrm{ej}, 53}^{1 / 2} T^{-1 / 2} \quad$ and $\quad \nu_{c, f}^{\mathrm{ob}} \approx 4.5 \times$ $10^{16} \mathrm{~Hz}\left(\epsilon_{B,-2}^{f}\right)^{-3 / 2} n_{0}^{-1} E_{\mathrm{ej}, 53}^{-1 / 2} T^{-1 / 2}(1+Y)^{-2}$. For the RS emission, we have $\nu_{m, r}^{\mathrm{ob}} \approx 1.0 \times 10^{18} \mathrm{Hzg}^{2}\left(\epsilon_{B}^{r}\right)^{1 / 2}\left(\epsilon_{e}^{r}\right)^{2} \times$ $\left(f_{e}^{r}\right)^{-2}\left(\Gamma_{\times}^{\prime}-1\right)^{2} n_{0}^{3 / 8} E_{\mathrm{ej}, 53}^{3 / 8} T^{-1 / 8}, \quad \nu_{c, r}^{\mathrm{ob}} \approx 4.5 \times$ $10^{13} \mathrm{~Hz}\left(\epsilon_{B}^{r}\right)^{-3 / 2} n_{0}^{-1} E_{\mathrm{ej}, 53}^{-1 / 2} T^{-1 / 2}(1+Y)^{-2}$. Basically, the observed cooling frequencies of RS and FS emissions are equal if microphysical parameters are similar, while the observed injection frequency of the RS emission is smaller than that of the FS emission by a factor of $\Gamma_{\times}^{2}$.

We also consider the synchrotron self-absorption process. The optical thickness for synchrotron self-absorption can be approximated by (see, e.g., [61]),

$$
\begin{aligned}
\tau_{s a}(\varepsilon) \simeq & \frac{5 e}{4 \pi r_{\times}^{2} B_{\times} \gamma_{e, n}^{5}} f_{e} N_{e} \\
& \times\left\{\begin{array}{ll}
\left(\varepsilon / \varepsilon^{n}\right)^{-(5 / 3)} & \left(\varepsilon<\varepsilon^{n}\right) \\
\left(\varepsilon / \varepsilon^{n}\right)^{-((p+4) / 2)} & \left(\varepsilon^{n} \leq \varepsilon\right)
\end{array},\right.
\end{aligned}
$$

where

$$
\begin{aligned}
N_{e}^{f} & =\frac{4 \pi}{3-k} A r^{3-k}, \\
N_{e}^{r} & =\frac{E_{\mathrm{ej}}}{\Gamma_{0} m_{p} c^{2}}
\end{aligned}
$$

are the number of electrons energized by the FS and RS, respectively. $\quad \gamma_{e, n} \equiv \min \left(\gamma_{e, c}, \gamma_{e, m}\right), \quad \varepsilon^{n} \equiv \min \left(\varepsilon^{m}, \varepsilon^{c}\right)$, and we have assumed $\gamma_{e, n}>1$. The self-absorption energy is determined by $\tau_{s a}\left(\varepsilon^{s a}\right)=1$. Hence, we can obtain

$$
\varepsilon^{s a} \simeq \varepsilon^{n}\left\{\begin{array}{ll}
\tau_{s a}^{3 / 5}\left(\varepsilon=\varepsilon^{n}\right) & \left(\varepsilon<\varepsilon^{n}\right) \\
\tau_{s a}^{2 /(p+4)}\left(\varepsilon=\varepsilon^{n}\right) & \left(\varepsilon^{n} \leq \varepsilon\right)
\end{array} .\right.
$$


Generally, the observed self-absorption frequency in the RS is typically higher than that in the FS.

The comoving specific luminosity per unit energy at the injection energy or cooling energy is approximated by

$$
L_{\varepsilon, \max }=\frac{1}{2 \pi \hbar} f_{e} N_{e} \frac{\sqrt{3} e^{3} B}{m_{e} c^{2}} \phi_{p},
$$

where $\phi_{p}$ is an order-unity coefficient calculated by Wijers and Galama [62]. In this paper we use $\phi_{p} \approx 0.6$. For example, let us consider the case of the thick ejecta collid- ing into the ISM. The observed peak luminosity per unit energy from the FS emission is written as $L_{\varepsilon_{\mathrm{ob}}, \max }^{f}=1.3 \times$ $10^{58} \mathrm{~s}^{-1} f_{e}^{f} E_{\mathrm{ej}, 53}\left(\epsilon_{B,-2}^{f}\right)^{1 / 2} n_{0}^{1 / 2} T^{1 / 2}\left(\phi_{p} / 0.6\right)$, and that from the RS emission is expressed as $L_{\varepsilon_{\mathrm{ob}}, \max }^{r}=4.9 \times$ $10^{62} \mathrm{~s}^{-1} f_{e}^{r} E_{\mathrm{ej}, 53}^{5 / 4}\left(\epsilon_{B}^{r}\right)^{1 / 2} n_{0}^{1 / 4} \Gamma_{0,2.5}^{-1} T^{-1 / 4}\left(\phi_{p} / 0.6\right)$. The observed peak flux from the RS is typically larger by a factor of $\Gamma_{\times}$than that from the FS.

From the above equations, we can obtain photon spectra. We have for $\varepsilon^{s a}<\varepsilon^{m}<\varepsilon^{c}$ (the slow cooling regime),

$$
\frac{d n}{d \varepsilon}=n_{\varepsilon, \max } \begin{cases}\left(\varepsilon^{s a} / \varepsilon^{m}\right)^{-(2 / 3)}\left(\varepsilon / \varepsilon^{s a}\right)^{1} & \left(\varepsilon^{\min } \leqq \varepsilon \leqq \varepsilon^{s a}\right) \\ \left(\varepsilon / \varepsilon^{m}\right)^{-(2 / 3)} & \left(\varepsilon^{s a}<\varepsilon \leqq \varepsilon^{m}\right) \\ \left(\varepsilon / \varepsilon^{m}\right)^{-((p+1) / 2)} & \left(\varepsilon^{m}<\varepsilon \leqq \varepsilon^{c}\right) \\ \left(\varepsilon^{c} / \varepsilon^{m}\right)^{-((p+1) / 2)}\left(\varepsilon / \varepsilon^{c}\right)^{-((p+2) / 2)} & \left(\varepsilon^{c}<\varepsilon \leqq \varepsilon^{\max }\right)\end{cases}
$$

and for $\varepsilon^{s a}<\varepsilon^{c}<\varepsilon^{m}$ (the fast cooling regime),

$$
\frac{d n}{d \varepsilon}=n_{\varepsilon, \max }\left\{\begin{array}{ll}
\left(\varepsilon^{s a} / \varepsilon^{c}\right)^{-(2 / 3)}\left(\varepsilon / \varepsilon^{s a}\right)^{1} & \left(\varepsilon^{\min } \leqq \varepsilon \leqq \varepsilon^{s a}\right) \\
\left(\varepsilon / \varepsilon^{c}\right)^{-(2 / 3)} & \left(\varepsilon^{s a}<\varepsilon \leqq \varepsilon^{c}\right) \\
\left(\varepsilon / \varepsilon^{c}\right)^{-(3 / 2)} & \left(\varepsilon^{c}<\varepsilon \leqq \varepsilon^{m}\right) \\
\left(\varepsilon^{m} / \varepsilon^{c}\right)^{-(3 / 2)}\left(\varepsilon / \varepsilon^{m}\right)^{-((p+2) / 2)} & \left(\varepsilon^{m}<\varepsilon \leqq \varepsilon^{\max }\right)
\end{array} .\right.
$$

Similarly we can obtain for $\varepsilon^{m}<\varepsilon^{s a}<\varepsilon^{c}$,

$$
\frac{d n}{d \varepsilon}=n_{\varepsilon, \max }\left\{\begin{array}{l}
\left(\varepsilon^{s a} / \varepsilon^{m}\right)^{-((p+1) / 2)}\left(\varepsilon^{m} / \varepsilon^{s a}\right)^{3 / 2}\left(\varepsilon / \varepsilon^{m}\right)^{1} \\
\left(\varepsilon^{s a} / \varepsilon^{m}\right)^{-((p+1) / 2)}\left(\varepsilon / \varepsilon^{s a}\right)^{3 / 2} \\
\left(\varepsilon / \varepsilon^{m}\right)^{-((p+1) / 2)} \\
\left(\varepsilon^{c} / \varepsilon^{m}\right)^{-((p+1) / 2)}\left(\varepsilon / \varepsilon^{c}\right)^{-((p+2) / 2)}
\end{array}\right.
$$

and for $\varepsilon^{c}<\varepsilon^{s a}<\varepsilon^{m}$ (where we do not consider the inhomogeneity [63]),

$$
\frac{d n}{d \varepsilon}=n_{\varepsilon, \max }\left\{\begin{array}{l}
\left(\varepsilon^{s a} / \varepsilon^{c}\right)^{-(3 / 2)}\left(\varepsilon^{c} / \varepsilon^{s a}\right)^{3 / 2}\left(\varepsilon / \varepsilon^{c}\right)^{1} \\
\left(\varepsilon^{s a} / \varepsilon^{c}\right)^{-(3 / 2)}\left(\varepsilon / \varepsilon^{s a}\right)^{3 / 2} \\
\left(\varepsilon / \varepsilon^{c}\right)^{-(3 / 2)} \\
\left(\varepsilon^{m} / \varepsilon^{c}\right)^{-(3 / 2)}\left(\varepsilon / \varepsilon^{m}\right)^{-((p+2) / 2)}
\end{array}\right.
$$

where

$$
n_{\varepsilon, \max }=\frac{L_{\varepsilon, \max }}{4 \pi r_{\times}^{2} \Gamma_{\times}^{2} c \varepsilon^{n}} .
$$

In order to demonstrate neutrino spectra from the RS, we adopt the following parameter sets.

ISM-tc: $\quad \Gamma_{0}=10^{2.5}, \quad E_{\mathrm{ej}}=4 \times 10^{53} \mathrm{ergs}, \quad \Delta_{0}=$ $4.5 \times 10^{11} \mathrm{~cm}, n=5 \mathrm{~cm}^{-3}, \epsilon_{B}^{r}=0.01, \epsilon_{e}^{r}=1 / 4, f_{e}^{r}=$ 1 , and $p=2.4$. This is one of the parameter sets representing the thick ejecta that collides into the ISM.

ISM-tn: $\quad \Gamma_{0}=10^{2}, \quad E_{\mathrm{ej}}=4 \times 10^{52} \mathrm{ergs}, \quad \Delta_{0}=$ $4.5 \times 10^{11} \mathrm{~cm}, n=0.5 \mathrm{~cm}^{-3}, \epsilon_{B}^{r}=0.01, \epsilon_{e}^{r}=1 / 4, f_{e}^{r}=$ 1 , and $p=2.4$. This is one of the parameter sets representing the thin ejecta that collides into the ISM.

ISM-e: $\quad \Gamma_{0}=300, \quad E_{\mathrm{ej}}=10^{55} \mathrm{ergs}, \quad \Delta_{0}=1.05 \times$ $10^{12} \mathrm{~cm}, n=1 \mathrm{~cm}^{-3}, \epsilon_{B}^{r}=0.001, \epsilon_{e}^{r}=0.04, f_{e}^{r}=1$, and $p=2.5$. This is one of the parameter sets representing the thin ejecta that collides into the ISM [17].
ISM-eb: $\quad \Gamma_{0}=300, \quad E_{\mathrm{ej}}=10^{54} \mathrm{ergs}, \quad \Delta_{0}=1.05 \times$ $10^{12} \mathrm{~cm}, n=1 \mathrm{~cm}^{-3}, \epsilon_{B}^{r}=0.2, \epsilon_{e}^{r}=0.1, f_{e}^{r}=1$, and $p=2.5$. This is one of the parameter sets representing the thick ejecta that collides into the ISM [16]. Note that the models ISM-e and ISM-eb express cases where the ejecta is energetic $E_{\mathrm{ej}} \gtrsim 10^{54} \mathrm{ergs}$. GRB 990123 is thought as such an energetic event. (Note that, if the ejecta carries the magnetic field directly from the central engine, $\epsilon_{B}^{r}$ can be significantly larger than $\epsilon_{B}^{f}$. A strong reverse shock is expected when the magnetic field is radiationally important but not yet dynamically important. GRB 990123 is thought to be one of such events [16].)

WIND-tc: $\Gamma_{0}=10^{2.5}, E_{\mathrm{ej}}=4 \times 10^{53} \mathrm{ergs}, \Delta_{0}=4.5 \times$ $10^{11} \mathrm{~cm}, A_{*}=1, \epsilon_{B}^{r}=0.01, \epsilon_{e}^{r}=1 / 4, f_{e}^{r}=1$, and $p=$ 2.4. This is one of the parameter sets representing the thick ejecta that collides into the windlike CBM.

WIND-tn: $\Gamma_{0}=10^{2}, E_{\mathrm{ej}}=4 \times 10^{52} \mathrm{ergs}, \Delta_{0}=4.5 \times$ $10^{11} \mathrm{~cm}, A_{*}=0.01, \epsilon_{B}^{r}=0.01, \epsilon_{e}^{r}=1 / 4$, and $p=2.4$. 
This is one of the parameter sets representing the thin ejecta that collides into the windlike CBM.

So far, we have assumed $f_{e}=1$. However, this might not be true [64]. We can consider cases with $f_{e} \ll 1$. If a small fraction of the electron population can have a significant part of the dissipated energy in the RS, the RS emission can appear in $\mathrm{x}$ rays [51]. The RS emission, which has usually been used for explanation of infrared/ optical flashes that are observed for only a small fraction of GRBs, might play an important role in the early afterglow phase. Recently, some authors proposed that the mysterious shallow decay emission can be explained by the RS emission [51,52]. In their models, the plateau shape can be achieved by requiring the appropriate distribution of Lorentz factors of the ejecta. It is assumed that Lorentz factors of the material, which is ejected during the last stages of source activity, decrease to small values of Lorentz factors. The head of the ejecta has larger Lorentz factors, while the tail of the ejecta has smaller Lorentz factors. In such cases, a long-lived RS is possible when it propagates into the stratified ejecta with decreasing Lorentz factors. In addition, this model also requires that the forward shock emission can be negligible at least in the early afterglow phase. The suppression of the FS emission might occur because the magnetic fields are too weak in the external medium and not sufficiently amplified [65], and/or the first-order Fermi acceleration mechanism is not so efficient. To reproduce the shallow decaying behavior, we need the detailed numerical modeling. This is beyond the scope of this paper because our goal is not to explain the shallow decay emission. For our purpose to estimate neutrino fluxes, it will be sufficient to consider the RS emission by the head of the ejecta. Assuming that the head of the ejecta carries the energy $E_{\text {ej }}^{h}=(1 / 3) E_{\text {ej }}$, we adopt the following parameter sets:

ISM-s: $\quad \Gamma_{0}=10^{2.5}, \quad E_{\mathrm{ej}}^{h}=\frac{4}{3} \times 10^{53} \mathrm{ergs}, \quad \Delta_{0}=$ $4.5 \times 10^{11} \mathrm{~cm}, n=5 \mathrm{~cm}^{-3}, \epsilon_{B}^{r}=1 / 4, \epsilon_{e}^{r}=1 / 4, f_{e}^{r}=$ 0.025 , and $p=2.4$. This is one of the parameter sets representing the thick ejecta that collides into the ISM.

WIND-s: $\Gamma_{0}=10^{2.5}, E_{\mathrm{ej}}^{h}=\frac{4}{3} \times 10^{53} \mathrm{ergs}, \Delta_{0}=4.5 \times$ $10^{11} \mathrm{~cm}, A_{*}=0.1, \epsilon_{B}^{r}=1 / 4, \epsilon_{e}^{r}=1 / 4, f_{e}^{r}=0.025$, and $p=2.4$. This is one of the parameter sets representing the thick ejecta that collides into the windlike CBM.

In order to predict neutrino fluxes, we have to set the amount of accelerated protons which no one knows from the first principle. In this paper, we just assume that the moderately efficient acceleration occurs and take $\epsilon_{\mathrm{acc}}=$ $1 / 4$, where $\epsilon_{\text {acc }} \equiv \zeta_{p}\left(1-\epsilon_{B}-\epsilon_{e}\right)$ and $\zeta_{p}$ is acceleration efficiency.

\section{B. The overlapping of prompt emission with the shocked region}

In the previous subsection, we have considered the RS emission under the original RS model, which typically predicts infrared/optical flashes. As previously noted, there is tentative evidence of the lack of infrared/optical flashes [46]. Several possible reasons have been suggested. First, the ejecta may be strongly magnetized [49]. Then, the hydrodynamical shock can become weak or there is no RS. Second, especially in the thin ejecta, the RS emission can be more suppressed than the earlier simplest estimations $[47,48]$. Such a semirelativistic RS may give a peak flux below one given by a FS. In addition, a pair-rich RS may be common [48]. Third, in the thick ejecta, the RS emission can be suppressed because RS electrons are rapidly cooled due to Compton scattering by photons from the prompt emission [50].

Now, we consider the third possibility, i.e., overlapping of the prompt emission with the shocked region. This overlapping will occur for the thick ejecta case, which can be expected for long GRBs we consider throughout this paper. The prompt emission which occurs due to internal dissipation can provide additional target photons for accelerated protons, so that more neutrinos can be produced via the photomeson production process. This possibility was suggested by Fan et al. [66] for the thick ejecta colliding into the windlike CBM. Here, we also study such possibilities including cases of the ejecta that collide with ISM in more detail. We assume $E_{\gamma}^{\text {iso }}=10^{53}$ ergs as the isotropic prompt emission energy. The averaged photon energy density within the ejecta at the crossing radius is given by

$$
U_{\gamma} \approx \frac{\Gamma^{2} E_{\gamma}^{\text {iso }}}{4 \pi r_{\times}^{2} \Delta_{0} \Gamma_{0}^{2}} .
$$

A spectrum of the prompt emission is well approximated by a broken power-law spectrum, which is

$$
\frac{d n}{d \varepsilon} \propto\left\{\begin{array}{ll}
\varepsilon^{-\alpha} & \left(\text { for } \varepsilon^{\min } \leq \varepsilon<\varepsilon^{b}\right) \\
\varepsilon^{-\beta} & \left(\text { for } \varepsilon^{b} \leq \varepsilon \leq \varepsilon^{\max }\right)
\end{array} .\right.
$$

The observed break energy is $\sim 250 \mathrm{keV}$, which corresponds to the break energy in the comoving frame, $\varepsilon^{b} \sim$ a few $\mathrm{keV}$. Hence, we set $\varepsilon^{b}=1 \mathrm{keV}$ and spectral indices to $\alpha=1, \beta=2.2$ similarly to our previous work [1]. We take the minimum energy as $1 \mathrm{eV}$ and the maximum energy as $10 \mathrm{MeV}$ in the comoving frame.

If such a photon flow due to the prompt emission cools down high energy electrons sufficiently, strong infrared/ optical flashes that are expected in the RS model can be suppressed [50]. In the model ISM-tc which represents the thick ejecta case, the cooling Lorentz factor of electrons $\gamma_{e, c}$ is significantly lowered due to Compton scattering by prompt photons. It leads to that the RS emission is not in the slow cooling regime but in the fast cooling regime.

In this paper, we consider the four parameter sets: ISMtc2, ISM-s2, WIND-tc2, and WIND-s2. Model parameters are the same as those in ISM-tc, ISM-s, WIND-tc, and WIND-s, respectively. But the overlapping effect due to the prompt emission is included. A target photon spectrum is given by a superposition of a prompt spectrum and a RS 
spectrum modified by the overlapping effect. Note that the high energy gamma-ray emission can be expected in this model $[50,66]$. These up-scattered photons can also contribute to the photomeson production process, but we can neglect such a population because of a smaller number of these high energy photons.

\section{The late prompt emission model}

The late internal dissipation may last longer than the duration of earlier internal dissipation that makes the prompt emission. For example, some of the flares are likely to be attributed to the late internal dissipations $[43,44]$. The flares typically happen hundreds of seconds after the trigger or earlier. In some cases, they occur around a day after the main burst. The amplitudes of the flares are usually larger than the underlying afterglow component by a factor of several, but can be much larger. Recent analyses show that the averaged radiation energy of flares is approximately $\sim 1 / 10$ of that of the prompt emission [44].

One of the leading models for such late internal dissipation is the late internal shock model (see, e.g., [67]). Lorentz factors of ejected subshells will be highly variable. If $\Gamma_{\mathrm{s}} \sim 10$ and $\Gamma_{\mathrm{f}} \sim 100$ are typical Lorentz factors of the slow and fast subshells, respectively, the Lorentz factor of the merged subshell can be expressed as $\Gamma_{0} \approx \sqrt{\Gamma_{\mathrm{f}} \Gamma_{\mathrm{s}}} \sim$ 30. The Lorentz factor of the internal shocks can be estimated by $\Gamma_{\mathrm{sh}} \approx\left(\Gamma_{f} / \Gamma_{s}+\Gamma_{s} / \Gamma_{f}\right) / 2 \sim$ a few. The typical collision radius is given by $r \approx 2 \Gamma_{0}^{2} c \delta t \approx$ $10^{15.3} \mathrm{~cm}\left(\Gamma_{0} / 15\right)^{2}[\delta t / 150(1+z) \mathrm{s}]$. The internal shocks are expected to be mildly relativistic shocks. If protons are accelerated efficiently in these shocks, neutrinos can appear through the photomeson production process. In our previous work [53], we predicted such high energy neutrino flashes from flares of GRBs.

On the other hand, the $\mathrm{x}$-ray emission in the shallow decay phase is often attributed to the external shock emission. However, Ghisellini et al. [45] recently suggested that this plateau phase for the x-ray emission may be due to the late prompt emission. In their model, this late prompt emission can be due to the same internal dissipation process as that for the early prompt emission but by subshells created at late times with smaller $\Gamma_{0}$ and much lower power. The radiation can be produced at distances relatively close to the central engine (even less than $r \sim$ $10^{13-14} \mathrm{~cm}$ ), in a different region where the subshells interact with the CBM [45].

Although this model can explain the chromatic behavior in early afterglows, it has not enabled us to explain the closure relations, which are expected in forward shock models and satisfied for the normal decay segment following the shallow decay segment. In spite of such a defect, there are indeed a couple of bursts that are likely to show the late prompt emission, marked by a sharp decay following an extended plateau with flickering. Such a striking behavior is seen in some bursts such as GRB 070110 [68].
If the shallow decay emission is attributed to the late prompt emission and late internal dissipation is due to late internal shocks or other models that allow a significant fraction of baryons to be accelerated up to sufficiently high energies, we can also expect high energy neutrino signals coincident with the shallow decay emission like the case of flares. The neutrino emission due to late internal dissipation itself would decay during the shallow and normal decay phases after the late prompt emission starts. Note that, physical conditions may be similar to those expected in flares and flares may be produced by a late shell, moving with a somewhat larger Lorentz factor than the shells created just earlier [45]. Therefore, we can use the same framework as that used in Murase and Nagataki [53] in order to evaluate high energy neutrino fluxes associated with the early $\mathrm{x}$-ray emission in the shallow decay phase under the late prompt emission model. In this paper, let us refer to neutrinos from both flares and the late prompt emission as neutrinos from the late prompt emission.

The photon energy density is given by

$$
U_{\gamma}=\frac{E_{\gamma, \mathrm{sh}}^{\mathrm{iso}}}{4 \pi \Gamma_{0} r^{2} l},
$$

where $l$ is the width of subshells and $E_{\gamma, \mathrm{sh}}^{\text {iso }}$ is the radiated energy from each subshell. $l$ is typically given by $l=r / \Gamma_{0}$, although it can be a smaller value. We assume the relatively small Lorentz factor, $\Gamma_{0} \sim$ a few $\times 10$. The emitted energy in the shallow decay phase is typically $\sim 1 / 10$ of that of the prompt emission [69]. Hence, we adopt the total isotropic radiation energy of the $\mathrm{x}$-ray emission in the shallow decay phase, $E_{\mathrm{LP}}^{\text {iso }} \equiv N E_{\gamma, \mathrm{sh}}^{\text {iso }}=10^{52}$ ergs, where $N$ is a number of collisions between ejected subshells. The shallow decay emission typically occurs at $T \sim$ $10^{3-4} \mathrm{~s}$, and a collision radius can be $r \sim 10^{13-16} \mathrm{~cm}$ under the late prompt emission model (and note that the expected variability time scale under the late internal shock model can be estimated by using $\left.\delta t \approx(1+z) r / 2 \Gamma_{0}^{2} c\right)$. Hence, we take $N \sim($ a few -100$)$.

For numerical calculations, we assume a broken powerlaw spectrum similar to the cases of the prompt emission, and we use Eq. (18). But we take $\varepsilon^{b}=(10-100) \mathrm{eV}$. We also set $\varepsilon^{\min }=0.1 \mathrm{eV}$ and $\varepsilon^{\max }=1 \mathrm{MeV}$.

Magnetic energy density is expressed as $U_{B} \equiv \xi_{B} U_{\gamma}$ where $\xi_{B} \approx \epsilon_{B} / \epsilon_{e}$. In this paper, we set $\xi_{B}=1$. Nonthermal proton energy density is parametrized as $U_{p} \equiv \xi_{\text {acc }} U_{\gamma}, \quad$ where $\quad \xi_{\text {acc }} \approx \epsilon_{\text {acc }} / \epsilon_{e}=\zeta_{p}\left(1-\epsilon_{B}-\right.$ $\left.\epsilon_{e}\right) / \epsilon_{e}$ is a nonthermal baryon loading factor, where $\zeta_{p}$ is the proton acceleration efficiency. The present acceleration theory cannot give this value from the first principle. Here, we assume that protons can be accelerated efficiently as is usually expected in supernova remnants and just adopt $\xi_{\text {acc }}=10$ as a fiducial value. If the proton distribution has $d n_{p} / d \varepsilon_{p} \propto \varepsilon_{p}^{-2}$ and the minimum energy of protons is a few $\times m_{p} c^{2}$, this fiducial value $\xi_{\text {acc }}=10$ means that 
the energy density of nonthermal protons with energy $\varepsilon_{p}$, $\varepsilon_{p}^{2}\left(\frac{d n_{p}}{d \varepsilon_{p}}\right)_{\varepsilon_{p}}$ is comparable to the radiation energy density of photons with energy $\varepsilon^{b},\left(\varepsilon^{b}\right)^{2}\left(\frac{d n}{d \varepsilon}\right)_{\varepsilon=\varepsilon^{b}}$. A similar assumption is often adopted in previous works [1-3,5]. If the nonthermal baryon loading factor could be larger, we expect higher neutrino fluxes, but no one obtains this value from the first principle. On the other hand, there is a hypothesis that ultra-high-energy cosmic rays (UHECRs) come from GRBs based on the internal shock model that will cause the usual prompt emission [2], which typically requires sufficiently large nonthermal baryon loading factors $\xi_{\text {acc }} \sim(50-100)$ [1] (although statements depend on the evaluation of the local GRB rate which has some uncertainties [70]). Motivated by this hypothesis, we also use $\xi_{\text {acc }}=50$ as the optimistic value in this paper.

We adopt the following parameter sets to estimate the neutrino flux from GRBs in the late prompt emission model:

LP0: $E_{\gamma, \mathrm{sh}}^{\mathrm{iso}}=10^{51.2} \mathrm{ergs}, \Gamma_{0}=15 r=10^{15.3} \mathrm{~cm}, \xi_{B}=$ 1 , and $\varepsilon^{b}=10 \mathrm{eV}$.

LP1: $E_{\gamma, \mathrm{sh}}^{\text {iso }}=10^{51} \mathrm{ergs}, \Gamma_{0}=10 r=10^{15} \mathrm{~cm}, \xi_{B}=1$, and $\varepsilon^{b}=100 \mathrm{eV}$.

LP2: $E_{\gamma, \mathrm{sh}}^{\text {iso }}=10^{50} \mathrm{ergs}, \Gamma_{0}=10 r=10^{14} \mathrm{~cm}, \xi_{B}=1$, and $\varepsilon^{b}=100 \mathrm{eV}$.

Note that the model LP0 corresponds to the model FUVray flare (A) used in Murase and Nagataki [53].

\section{NEUTRINO PRODUCTION IN GRBS}

We have assumed that the early afterglow emission comes from high energy electrons that are accelerated via some dissipation process such as shock dissipation. Protons also may be accelerated in internal and/or external shocks. If the first-order Fermi acceleration mechanism is realized, a proton spectrum can be written as

$$
\frac{d n_{p}}{d \varepsilon_{p}}=\frac{U_{p}}{\int_{\varepsilon_{p}^{\min }}^{\varepsilon^{\max }} d \varepsilon_{p}\left(\varepsilon_{p} \frac{d n_{p}}{d \varepsilon_{p}}\right)} \varepsilon_{p}^{-p},
$$

where $p$ is the spectral index. Its value is typically $p \approx 2$ in the nonrelativistic shock diffusive acceleration theory. In the ultrarelativistic shock limit, $p \approx 2.2$ is obtained assuming the isotropic diffusion in the downstream [71,72]. In this paper, we adopt $p=2$ for the proton spectrum. $U_{p}$ is the energy density of nonthermal protons, which is given by $U_{p}=\xi_{\text {acc }} U_{\gamma}$ in the late prompt emission model while by $U_{p}=\epsilon_{\mathrm{acc}}\left(E_{\mathrm{ej}} / 4 \pi r_{\times}^{2} \Gamma_{\times}^{2} \Delta_{\times}\right)$. (Note that the neutrino background can be calculated, given the GRB rate history. For this purpose, we also use the different normalization based on the observed UHECR flux. See the end of this subsection.) The minimum energy of protons would be $\varepsilon_{p}^{\min } \sim$ a few $\times \Gamma_{\text {rel }} m_{p} c^{2}$, although the exact value is unknown. Here, $\Gamma_{\text {rel }}$ is the relative Lorentz factor, which is $\Gamma_{\text {rel }}=\Gamma^{\prime}$ for the RS or $\Gamma_{\text {rel }}=\Gamma_{\text {sh }}$ for the internal shocks. Here, we take $\varepsilon_{p}^{\min }=10 \mathrm{GeV}$, although the accurate value is irrelevant for the resulting spectra. For the FS, the proton energy in the observer frame is given by $E_{p}^{\min } \sim$ a few $\times$ $\Gamma m_{p} c^{2}$.

The maximum energy of cosmic-ray nuclei is determined by several criteria. One of the necessary conditions is obtained by comparing the Larmor radius of cosmic-ray nuclei with the size of the acceleration region [73,74]. In cases we consider, this criterion corresponds to comparing the acceleration time scale $t_{\text {acc }}$ with the dynamical time scale $t_{\mathrm{dyn}}$. In addition, the maximum energy of particles is limited by various cooling processes and diffusive losses of them. Corresponding criteria are obtained by comparing the acceleration time scale with various cooling time scales (the synchrotron cooling time scale $t_{\mathrm{syn}}$, adiabatic cooling time scale $t_{\mathrm{ad}}$, and so on) and with the escape time scale due to particle diffusion $t_{\mathrm{esc}}[1,30,73]$. For cosmic-ray nuclei to be accelerated, all the above criteria should be satisfied. As an example, let us consider the first criterion and estimate the possible maximum energy of protons accelerated in the FS for the thick ejecta colliding into ISM. We have [75]

$$
\begin{aligned}
E_{p}^{\max } & \approx Z e \Gamma_{\times} B^{\mathrm{ISM}} r_{\times} \\
& \approx 5.1 \times 10^{15} \mathrm{eVZB} B_{-6}^{\mathrm{ISM}} E_{\mathrm{ej}, 53}^{3 / 8} n_{0}^{-3 / 8} T_{1}^{1 / 8},
\end{aligned}
$$

where $B^{\mathrm{ISM}}$ is the strength of the upstream magnetic field. From the above equation, we can expect that protons cannot be accelerated up to ultra high energies at the FS by the first-order Fermi acceleration mechanism [65,75]. Although the second-order Fermi acceleration might allow protons to be accelerated up to ultra high energies [76], we consider only the first-order Fermi acceleration mechanism for the FS in this paper. From Eq. (21), we can see that neutrinos, which are produced by the photomeson production process in the FS, will be negligible. Corresponding to Eq. (21), the maximum energy of protons accelerated in the $\mathrm{RS}$ is written as

$$
E_{p}^{\max } \approx Z e B_{\times}^{r} r_{\times} \approx 2.0 \times 10^{21} \mathrm{eVZ} \epsilon_{B}^{1 / 2} E_{\mathrm{ej}, 53}^{3 / 8} n_{0}^{1 / 8} T_{1}^{1 / 8} .
$$

Therefore, protons can be accelerated up to ultra high energies at the RS by the first-order acceleration mechanism if other cooling processes are not important. On the other hand, in the late prompt emission model, the UHECR production is typically impossible. Note that the photomeson cooling process can be important in very high energies in this model [53]. We treat various cooling time scales properly in our numerical results that are shown later (see Appendix A).

Sufficiently accelerated protons can interact with target photons via the photomeson production process and produce high energy pion and muons. Neutrinos are produced via the decay of $\pi^{ \pm} \rightarrow \mu^{ \pm}+\nu_{\mu}\left(\bar{\nu}_{\mu}\right) \rightarrow e^{ \pm}+\nu_{e}\left(\bar{\nu}_{e}\right)+$ $\nu_{\mu}+\bar{\nu}_{\mu}$. The neutrino production efficiency is repre- 
sented by the photomeson production efficiency $f_{p \gamma} \equiv$ $t_{\mathrm{dyn}} / t_{p \gamma}$ (see Appendix A). For example, let us consider the thick ejecta colliding into the ISM. In such cases, we can approximately obtain $[2,27,53]$,

$$
f_{p \gamma} \simeq 0.088 \frac{L_{b, 48}}{r_{\times, 16} \Gamma_{\times, 2}^{2} E_{10 \mathrm{eV}}^{b}}\left\{\begin{array}{ll}
\left(E_{p} / E_{p}^{b}\right)^{\beta-1} & \left(E_{p}<E_{p}^{b}\right) \\
\left(E_{p} / E_{p}^{b}\right)^{\alpha-1} & \left(E_{p}^{b} \leq E_{p}\right)
\end{array},\right.
$$

where $E^{b}$ is the observed break energy which is either of $\varepsilon_{\mathrm{ob}}^{c}$ or $\varepsilon_{\mathrm{ob}}^{m}$ or $\varepsilon_{\mathrm{ob}}^{s a}$, and $L_{b}$ is the observed luminosity at the break energy. $E_{p}^{b} \simeq 0.5 \bar{\varepsilon}_{\Delta} m_{p} c^{2} \Gamma_{\times}^{2} / E^{b}$ is the proton break energy, where $\bar{\varepsilon}_{\Delta}$ is around $0.3 \mathrm{GeV}$. For example, $E^{b}=$ $10 \mathrm{eV}$ and $\Gamma_{\times}=100$ lead to $E_{p}^{b} \sim 10^{20} \mathrm{eV}$. In Eq. (23) we do not include the effect of multipion production which is not important for the RS emission.

Next, let us consider the overlapping of the prompt emission with the shocked region. We can obtain (including the effect of multipion production which is moderately important in very high energies) [53],

$$
f_{p \gamma} \simeq 1.7 \times 10^{-3} \frac{\Gamma^{2} E_{\gamma, 53}^{\text {iso }}}{r_{\times, 16} \Delta_{0,12} \Gamma_{0,2.5}^{2} E_{100 \mathrm{keV}}^{b}}\left\{\begin{array}{l}
\left(E_{p} / E_{p}^{b}\right)^{\beta-1} \\
\left(E_{p} / E_{p}^{b}\right)^{\alpha-1}
\end{array} .\right.
$$

From Eq. (24), we expect that photomeson production is not so efficient when the CBM is the ISM. If the CBM is windlike, it becomes more efficient [66].

Finally, let us consider the late prompt emission model. The physical conditions will be similar to those of flares. From Eq. (3) in Murase and Nagataki [53], we have

$$
f_{p \gamma} \simeq 5.2 \frac{E_{\gamma, \mathrm{sh}, 50.5}^{\mathrm{iso}}}{r_{14.5}^{2} E_{1 \mathrm{keV}}^{b}}\left\{\begin{array}{l}
\left(E_{p} / E_{p}^{b}\right)^{\beta-1} \\
\left(E_{p} / E_{p}^{b}\right)^{\alpha-1}
\end{array} .\right.
$$

From Eq. (25), we can expect that almost all the protons that are accelerated to sufficiently high energies will be depleted due to photomeson production. The above approximate evaluation by using Eqs. (23)-(25) is in good agreement with numerical results.

We treat photomeson production in detail and calculate neutrino spectra numerically. Generally, cooling processes of pions and muons are important. Of course, our numerical calculations take into account them, but the analytical consideration is convenient. Hence, we briefly review the neutrino emission process here. More detailed discussions can be found in e.g., Rachen and Mészáros [73].

One of the important cooling processes is synchrotron cooling of pions and muons. The synchrotron break for neutrinos from pions/muons is determined by $t_{\pi / \mu \text {,syn }}=$ $\gamma_{\pi / \mu} \tau_{\pi / \mu}$, where $\tau_{\pi / \mu}$ is the mean lifetimes of pions and muons. For neutrinos from pions and muons, we obtain

$$
\begin{aligned}
E_{\nu}^{\pi, s} & \approx \frac{1}{4} \Gamma \sqrt{\frac{6 \pi m_{\pi}^{5} c^{5}}{\sigma_{\mathrm{T}} m_{e}^{2} B^{2} \tau_{\pi}}}, \\
E_{\nu}^{\mu, s} & \approx \frac{1}{3} \Gamma \sqrt{\frac{6 \pi m_{\mu}^{5} c^{5}}{\sigma_{\mathrm{T}} m_{e}^{2} B^{2} \tau_{\mu}}} .
\end{aligned}
$$

Above the synchrotron break, a neutrino spectrum is suppressed by $t_{\pi / \mu \text {,syn }} / t_{\pi / \mu}$. The adiabatic break for neutrinos from pions/muons is determined by $t_{\pi / \mu \text {,ad }}=\gamma_{\pi / \mu} \tau_{\pi / \mu}$. We have

$$
\begin{aligned}
E_{\nu}^{\pi, a} & \approx \frac{1}{4} \Gamma \frac{t_{\pi}}{\tau_{\pi}} m_{\pi} c^{2}, \\
E_{\nu}^{\mu, a} & \approx \frac{1}{3} \Gamma \frac{t_{\mu}}{\tau_{\mu}} m_{\mu} c^{2} .
\end{aligned}
$$

Above the adiabatic break, a neutrino spectrum is suppressed by $t_{\pi / \mu \text {,ad }} / t_{\pi / \mu}$.

Now, we can obtain approximate neutrino spectra of $\left(\nu_{\mu}+\bar{\nu}_{\mu}\right)$. For $E_{\nu}^{b}<E_{\nu}^{s}\left(<E_{\nu}^{a}\right)$, we obtain

$$
E_{\nu}^{2} \frac{d N_{\nu}}{d E_{\nu}} \approx \frac{1}{4} f_{p \gamma} E_{p}^{2} \frac{d N_{p}}{d E_{p}}\left\{\begin{array}{l}
\left(E_{\nu} / E_{\nu}^{b}\right)^{\beta-1} \\
\left(E_{\nu} / E_{\nu}^{b}\right)^{\alpha-1} \\
\left(E_{\nu}^{s} / E_{\nu}^{b}\right)^{\alpha-1}\left(E_{\nu} / E_{\nu}^{s}\right)^{\alpha-3}
\end{array} .\right.
$$

For $E_{\nu}^{b}<E_{\nu}^{a}<E_{\nu}^{s}$, we have

$$
\begin{aligned}
E_{\nu}^{2} \frac{d N_{\nu}}{d E_{\nu}} \approx & \frac{1}{4} f_{p \gamma} E_{p}^{2} \frac{d N_{p}}{d E_{p}} \\
& \times\left\{\begin{array}{l}
\left(E_{\nu} / E_{\nu}^{b}\right)^{\beta-1} \\
\left(E_{\nu} / E_{\nu}^{b}\right)^{\alpha-1} \\
\left(E_{\nu}^{a} / E_{\nu}^{b}\right)^{\alpha-1}\left(E_{\nu} / E_{\nu}^{a}\right)^{\alpha-2} \\
\left(E_{\nu}^{a} / E_{\nu}^{b}\right)^{\alpha-1}\left(E_{\nu}^{s} / E_{\nu}^{a}\right)^{\alpha-2}\left(E_{\nu} / E_{\nu}^{s}\right)^{\alpha-3}
\end{array}\right.
\end{aligned}
$$

Note that the above two approximate expressions are valid as long as $f_{p \gamma}<1$. Although more general expressions can be obtained, we do not show them here. We can obtain general neutrino more quantitatively by grace of numerical calculations.

It is important to see a lot of GRB events, because the detection of neutrinos signals from one GRB is generally not easy $[1,4]$. We can calculate the neutrino background assuming the GRB rate history (see Appendix B). In this paper, we adopt two different normalizations of the proton flux. One is the normalization by the observed GRB rate and isotropic energy with the fixed acceleration efficiency. As noted before, we adopt $\epsilon_{\text {acc }}=1 / 4$ for the RS model and $\xi_{\text {acc }}=10$ for the late prompt emission model as fiducial values, respectively. The other normalization is based on the hypothesis that observed UHECRs (whose flux is $E_{p}^{2} d \dot{N}_{p} / d E_{p}(z=0) \approx 0.65 \times$ $10^{44} \mathrm{ergs} \mathrm{Mpc}^{-3} \mathrm{yr}^{-1}$ ) come from GRBs. When we adopt this normalization, we simply change the normalization factor just for simplicity. For more detailed calculations, 
proton spectra after the propagation in the Universe should be fitted to the observed UHECR flux, and we have to take care of affections to target photon spectra by the change of the released energy per GRB according to the assumed local GRB rate. In the RS model we expect that UHECR production is possible, so that neutrino fluxes from the RS should generally satisfy the Waxman and Bahcall (WB) bound [77] because of $f_{p \gamma} \lesssim 1$ typically. For the late prompt emission model, we adopt $\xi_{\text {acc }}=50$ as an optimistic value, as is noted in the previous section. Note that neutrino fluxes in this model do not have to satisfy the WB bound generally (rather, it should be constrained by the more general bound [78]). This is because protons are usually not accelerated up to ultra high energies and we can expect $f_{p \gamma} \gtrsim 1$ in this model.

\section{NUMERICAL RESULTS}

\section{A. Neutrino spectra from GRB early afterglows}

We need to evaluate proton cooling time scales which include the photohadronic cooling calculated by Geant 4 . In Fig. 1, we show the acceleration time scale and proton cooling time scales for the model ISM-e which represents the case of energetic ejecta that is expected in GRB 990123. In this case, the most important cooling process is adiabatic cooling. The maximum energy is determined by Eq. (22). For the ISM cases such as the models ISM-tc and ISM-tn, the adiabatic time scale is typically the most important. For the windlike CBM cases, synchrotron cooling can be more important. On the other hand, photohadronic cooling can be a dominant cooling process in the late prompt emission model, as shown in Murase and Nagataki [53]. Synchrotron cooling and adiabatic cooling can also be important.

In the case of the prompt emission, the effect of pion multiplicity and proton inelasticity is moderately important

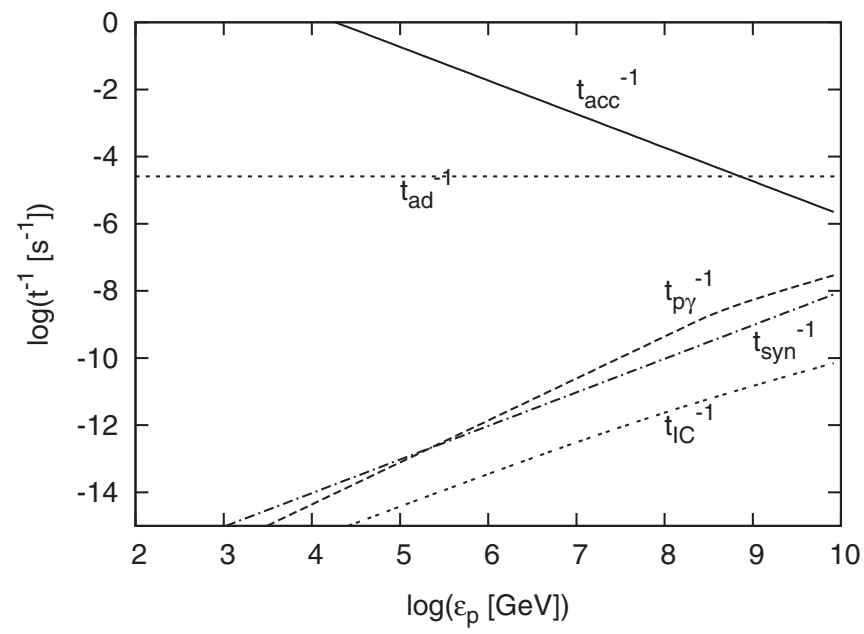

FIG. 1. The acceleration time scale and various cooling time scales of protons for the model ISM-e. The energy scale is measured in the comoving frame. in the sufficiently high energies. This comes from the fact that more and more target photons can interact with protons via double and multipion production, as the energy of incident protons becomes high. As a result, the photohadronic cooling time scale increases with the energy more than expected in the $\Delta$-resonance approximation [1]. Similar things can be applied when we consider the overlapping effect of the prompt emission and the late prompt emission model. This effect is more important for a flatter photon spectrum as demonstrated in the previous paper. On the other hand, the RS emission $(\alpha \sim(1.5-1.7)$ (for $\varepsilon<$ $\max \left[\varepsilon^{m}, \varepsilon^{c}\right]$ ) and $\beta \sim 2.2$ (for $\varepsilon>\max \left[\varepsilon^{m}, \varepsilon^{c}\right]$ )) has a steeper spectrum than that of the prompt emission ( $\alpha=$ 1 and $\beta=2.2$ ). Therefore, the multiplicity effect is less important in the case of the RS emission and the $\Delta$-resonance approximation is a good approximation. In fact, Eq. (23) is in good agreement with numerical results.

By executing numerical calculations, we can obtain neutrino spectra (see Appendix A). The adopted parameter sets are summarized in Tables I and II. First, we show the neutrino energy fluence from one GRB event. The neutrino energy fluence can be evaluated by

$$
E_{\nu}^{2} \phi_{\nu}=\frac{(1+z)}{4 \pi d_{L}^{2}} \Gamma \varepsilon_{\nu}^{2} \frac{d N_{\nu}}{d \varepsilon_{\nu}},
$$

where $\varepsilon_{\nu}$ is the neutrino energy in the comoving frame and $d_{L}$ is the luminosity distance to the source for a given cosmology. Throughout the paper, we assume the $\Lambda \mathrm{CDM}$ universe with $\Omega_{m}=0.3, \Omega_{k}=0, \Omega_{\Lambda}=0.7$, and $H_{0}=$ $71 \mathrm{~km} \mathrm{~s}^{-1} \mathrm{Mpc}^{-1}$.

TABLE I. The adopted parameter sets in the reverse-forward shock model. Note that the models, where the overlapping effect due to the prompt emission is included, are referred to as ISMtc2, ISM-s2, WIND-tc2, and WIND-s2. The isotropic prompt emission energy $E_{\gamma}^{\text {iso }}=10^{53}$ ergs is assumed in these four parameter sets.

\begin{tabular}{lcccllll}
\hline \hline Model & $\Gamma_{0}$ & $E_{\mathrm{ej}}[\mathrm{ergs}]$ & $\Delta_{0}[\mathrm{~cm}]$ & $n\left(A_{*}\right)$ & $\epsilon_{B}^{r}$ & $\epsilon_{e}^{r}$ & $f_{e}^{r}$ \\
\hline ISM-tc & $10^{2.5}$ & $4 \times 10^{53}$ & $4.5 \times 10^{11}$ & 5 & 0.01 & 0.25 & 1 \\
ISM-tn & $10^{2}$ & $4 \times 10^{52}$ & $4.5 \times 10^{11}$ & 0.5 & 0.01 & 0.25 & 1 \\
ISM-s & $10^{2.5}$ & $4 \times 10^{53}$ & $4.5 \times 10^{11}$ & 5 & 0.25 & 0.25 & 0.025 \\
ISM-e & 300 & $10^{55}$ & $1.05 \times 10^{12}$ & 1 & 0.001 & 0.04 & 1 \\
ISM-eb & 300 & $10^{54}$ & $1.05 \times 10^{12}$ & 1 & 0.2 & 0.1 & 1 \\
WIND-tc & $10^{2.5}$ & $4 \times 10^{53}$ & $4.5 \times 10^{11}$ & 1 & 0.01 & 0.25 & 1 \\
WIND-tn & $10^{2}$ & $4 \times 10^{52}$ & $4.5 \times 10^{11}$ & 0.01 & 0.01 & 0.25 & 1 \\
WIND-s & $10^{2.5}$ & $4 \times 10^{53}$ & $4.5 \times 10^{11}$ & 0.1 & 0.25 & 0.25 & 0.025 \\
\hline \hline
\end{tabular}

TABLE II. The adopted parameter sets in the late prompt emission model.

\begin{tabular}{lccccc}
\hline \hline Model & $\Gamma_{0}$ & $E_{\gamma, \mathrm{sh}}^{\text {iso }}[\mathrm{ergs}]$ & $r[\mathrm{~cm}]$ & $\xi_{B}$ & $E^{b /}[\mathrm{keV}]$ \\
\hline LP0 & 15 & $10^{51.2}$ & $10^{15.3}$ & 1 & 0.15 \\
LP1 & 10 & $10^{51}$ & $10^{15}$ & 1 & 1 \\
LP2 & 10 & $10^{50}$ & $10^{14}$ & 1 & 1 \\
\hline \hline
\end{tabular}


KOHTA MURASE

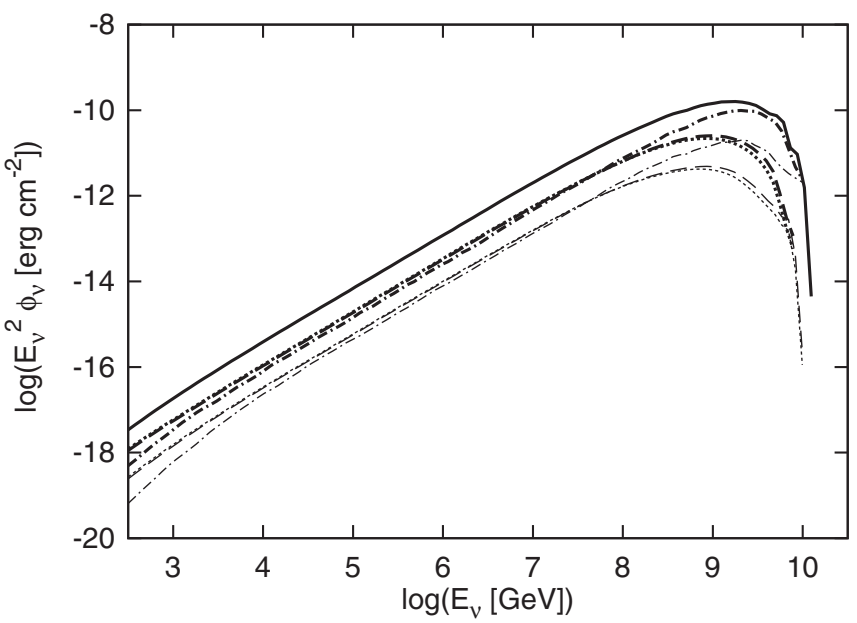

FIG. 2. Neutrino fluences from one energetic GRB event at $z=1.6$. Neutrinos are produced by the decay of pion and muon whose origins are single-pion production (thick lines), and double and multipion production (thin lines). The model ISMe is used. We show fluences of muon neutrinos $\left(\nu_{\mu}+\bar{\nu}_{\mu}\right)$ from pion decay (dot-dashed lines), muon neutrinos $\left(\nu_{\mu}+\bar{\nu}_{\mu}\right)$ from muon decay (dashed-lines), and electron neutrinos $\left(\nu_{e}+\bar{\nu}_{e}\right)$ from muon decay (dotted lines). The total neutrino fluence is also shown by the thick solid line. The normalization of the proton flux is given by $\epsilon_{\mathrm{acc}}=1 / 4$ and neutrino oscillation is not taken into account.

In Fig. 2, we show neutrino energy fluences for the model ISM-e. Even for prompt neutrino bursts predicted under the internal shock model, it is difficult to see high energy signals from one GRB event by IceCube, which typically requires $E_{\nu}^{2} \phi_{\nu} \gtrsim 10^{-4} \mathrm{erg} \mathrm{cm}^{-2}$ for $0.1 \mathrm{PeV}$ neutrinos [4]. Therefore, it is much more difficult to see neutrino afterglows under the original RS model because the smaller number of neutrinos with higher energies is expected in this model.

We comment on the effect of double and multipion production to neutrino spectra. In the case of the prompt emission, this effect can become moderately important and enhance the neutrino fluence in the very high energies [1]. But this effect is hardly important in the case of the RS emission, as seen in Fig. 2.

In Fig. 3, we show resulting spectra in the RS model with the ISM. For all the models, we hardly expect neutrino signals by IceCube. As an example, in the model ISM-tc, we have small photomeson production efficiency $f_{p \gamma} \sim$ $5 \times 10^{-3}$ (at $\varepsilon_{p} \approx 10^{8.5} \mathrm{GeV}$ ). In the model ISM-tn, smaller $E_{\text {ej }}$ and smaller $f_{p \gamma} \sim 5 \times 10^{-4}$ (at $\varepsilon_{p} \approx$ $10^{9} \mathrm{GeV}$ ) lead to much smaller neutrino fluences. In the original analytical prediction [27], the neutrino energy fluence increases as neutrino energy is high. But, the more realistic spectra show the suppressed behavior around $10^{9} \mathrm{GeV}$. This is just because protons have the finite maximum energy so that we see the cooling effect of pions and muons in the highest energies. While the fluence of highest energy neutrinos is suppressed above $E_{\nu} \sim$
PHYSICAL REVIEW D 76, 123001 (2007)

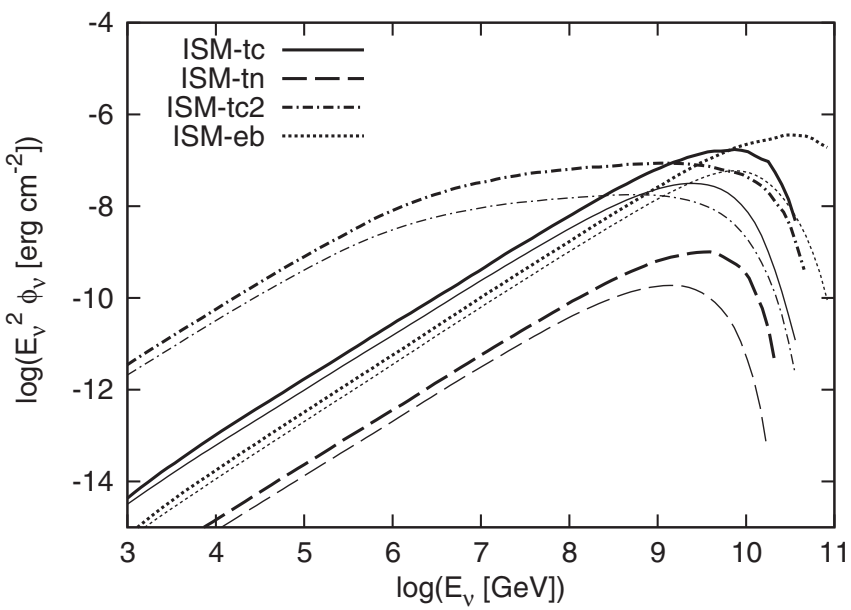

FIG. 3. Muon-neutrino $\left(\nu_{\mu}+\bar{\nu}_{\mu}\right)$ fluences (thick lines) and electron-neutrino $\left(\nu_{e}+\bar{\nu}_{e}\right)$ fluences (thin lines) from one GRB event at $z=0.1$. The RS models with the ISM are used. Note that the normalization of the proton flux is given by $\epsilon_{\mathrm{acc}}=1 / 4$ and neutrino oscillation is not taken into account.

$10^{9} \mathrm{GeV}$ compared to the analytical result, we can see $E_{\nu}^{2} \phi_{\nu} \propto E_{\nu}^{1.2}$ for $E_{\nu} \lesssim 10^{9} \mathrm{GeV}$, which is in good agreement with the analytical result. In the model ISM-eb, we can see that neutrino spectra are extended to higher energies. This is just because the maximum energy of protons in this model, where large $\epsilon_{B}^{r}=0.2$ is taken, is higher than in the models ISM-tc and ISM-tn (by a factor of $\sim 5$ larger than in the model ISM-tc).

In the model ISM-tc2, we consider overlapping of the prompt emission. Once this is taken into account, neutrino fluence is greatly enhanced by 2 or 3 orders of magnitude. Therefore, the effect of this overlapping is important. Nevertheless, the detection by IceCube will be very difficult unless a burst with $E_{\text {ej }} \sim 10^{54-55}$ ergs occurs at $\sim 10 \mathrm{Mpc}$.

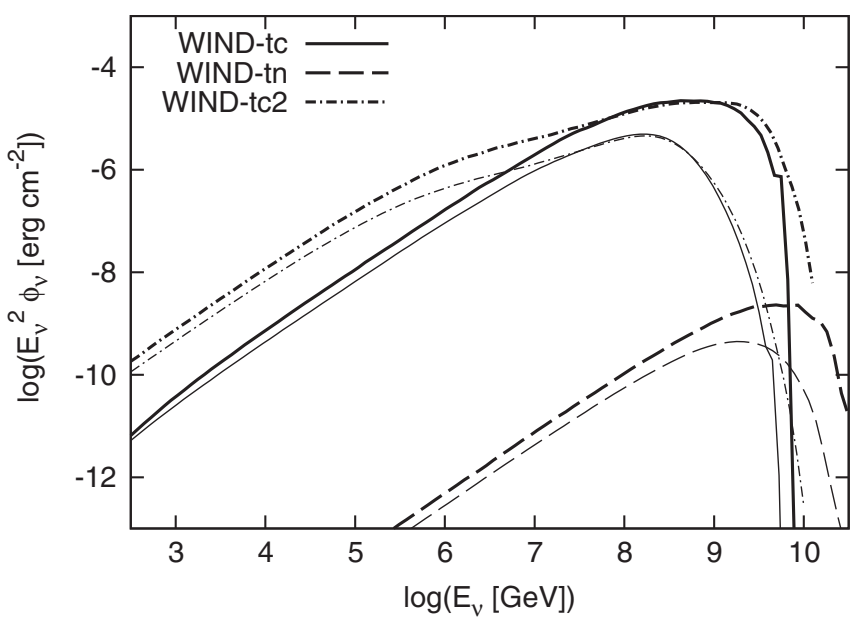

FIG. 4. The same as Fig. 3, but for the RS models with the windlike CBM. 
As shown in Fig. 4, we can expect that the neutrino emission occurs efficiently in the model WIND-tc, where we obtain $f_{p \gamma} \gtrsim 1$ (for $\varepsilon_{p} \gtrsim 10^{8} \mathrm{GeV}$ ). It is because the crossing radius in the model WIND-tc is smaller than that in the models with the ISM. Below $E_{\nu} \sim 10^{8} \mathrm{GeV}$, we can see $E_{\nu}^{2} \phi_{\nu} \propto E_{\nu}^{1.2}$ (note that the break corresponding to $\varepsilon_{\mathrm{ob}}^{m} \approx 8.3 \mathrm{eV}$ is $E_{\nu}^{m} \sim 10^{9.8} \mathrm{GeV}$, which we cannot see because the fluence is saturated at energy such that $f_{p \gamma} \sim$ 1). While the spectrum of the model WIND-tc is in the fast cooling regime, that of the model WIND-tn is in the slow cooling regime. We cannot expect high photomeson production efficiency in the latter model. We have $f_{p \gamma} \sim 3 \times$ $10^{-4}$ (at $\varepsilon_{p} \approx 10^{8.5} \mathrm{GeV}$ ) due to the smaller $E_{\text {ej }}$ and larger crossing radius.

If we consider overlapping of the prompt emission for the models with the windlike CBM, neutrino fluence can be higher by 1 order of magnitude due to additional target photons from the prompt emission.

In Fig. 5, neutrino spectra are calculated under the RS model with the higher $\epsilon_{B}$ and smaller $f_{e}$. We choose such parameters based on the modified RS model that explains the shallow decay emission [51,52]. We calculate for the RS produced by the head of the ejecta, although the RS emission would continue by the slower tail of the ejecta. Although additional protons supplied by the tail of the ejecta may produce neutrinos, our estimation on neutrino fluences would not be changed so much up to a factor. Target photon spectra in both models ISM-s and WIND-s, are expected in the fast cooling regime at the crossing time of the head of the ejecta. In this RS model, significant energy of the RS emission is radiated as $\mathrm{x}$-ray photons that can interact with protons with energy $E_{p} \sim 10^{9} \mathrm{GeV}$, which produce neutrinos with $E_{\nu} \sim 5 \times 10^{7} \mathrm{GeV}$. For the model ISM-s, we have the photomeson efficiency $f_{p \gamma} \sim 0.01$ (at $\varepsilon_{p} \approx 10^{8.5} \mathrm{GeV}$ ), which is higher than

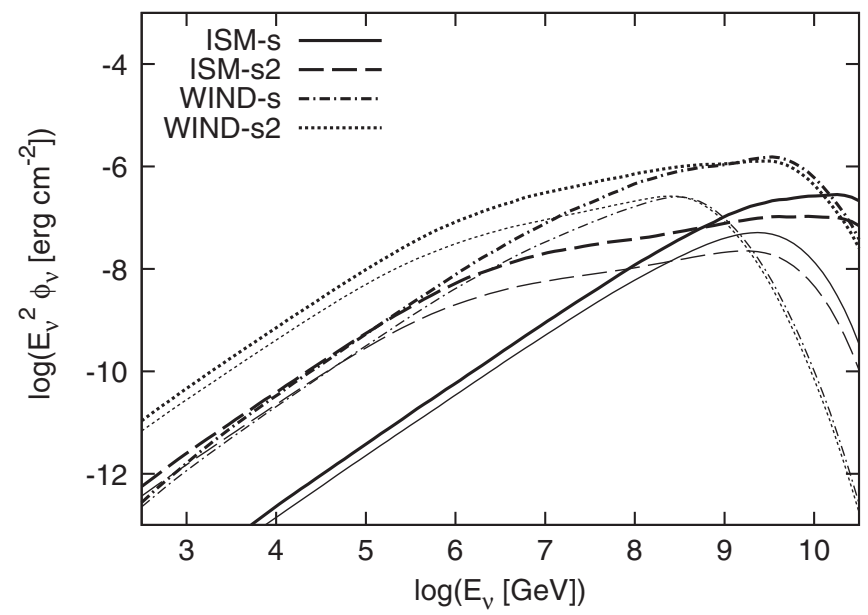

FIG. 5. The same as Fig. 3, but model parameters are chosen, motivated by the modified RS model explaining the shallow decay emission.

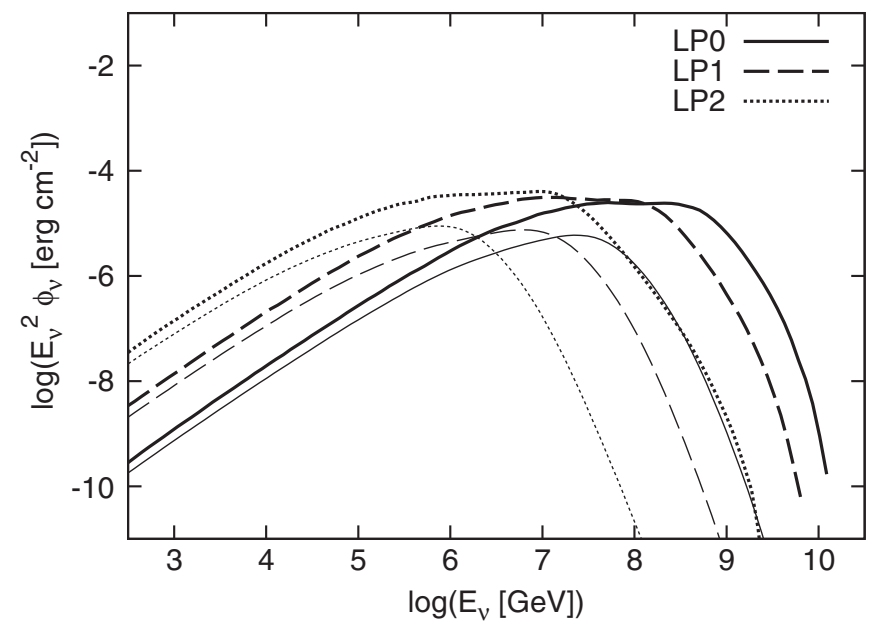

FIG. 6. The same as Fig. 3, but for the models based on the late prompt emission model. The normalization of the proton flux is given by $\xi_{\mathrm{acc}}=10$.

that in the model ISM-tc. It is because photons that can interact with protons increase in this RS model due to its higher injection energy $\varepsilon_{\mathrm{ob}}^{m} \sim 0.3 \mathrm{keV}$. On the other hand, in the model WIND-s, we obtain $f_{p \gamma} \sim 0.1$ (at $\varepsilon_{p} \approx$ $10^{9} \mathrm{GeV}$ ), which is smaller than that in the model WIND-tc. This is because most of the radiated energy is emitted as photons with $\varepsilon_{\mathrm{ob}}^{m} \sim 7 \mathrm{keV}$ which is higher than that in the original RS model. After all, it reduces the number of target photons that can interact with protons. Corresponding to $\varepsilon_{\mathrm{ob}}^{m} \sim 7 \mathrm{keV}$, we expect the break at $E_{\nu}^{m} \sim 10^{7} \mathrm{GeV}$, which can be seen in Fig. 5. We can also find that overlapping of the prompt emission enhances neutrino fluence greatly in Fig. 5.

In Fig. 6, we show results obtained under the late prompt emission model. For all the three models, we obtain $f_{p \gamma} \gtrsim$ 1. Expected muon events from one GRB event is $N_{\mu} \sim$ (0.3-0.6) events for the model LP2. Therefore, if bright GRBs occur, we have possibilities to detect neutrino signals from early afterglows in the late prompt emission model. Because most of the sufficiently high energy protons are depleted in these three models, we see the similar level of the energy fluence. In our models, the magnetic field strength is stronger than that in the RS model. Hence, neutrino spectra are suppressed in the highest energies due to cooling processes of pions and muons. (Note that the model LP0 has the same parameter set used in Murase and Nagataki [53], but we have the higher energy fluence by a factor because we showed the energy fluence per internal collision in the previous paper.)

\section{B. GRB neutrino background}

Since it is difficult to see neutrino signals from one GRB event, we have to observe many GRBs. Because we can find a good fraction of GRBs by space satellites, we expect neutrino signals that are coincident with bursts seen by 
KOHTA MURASE

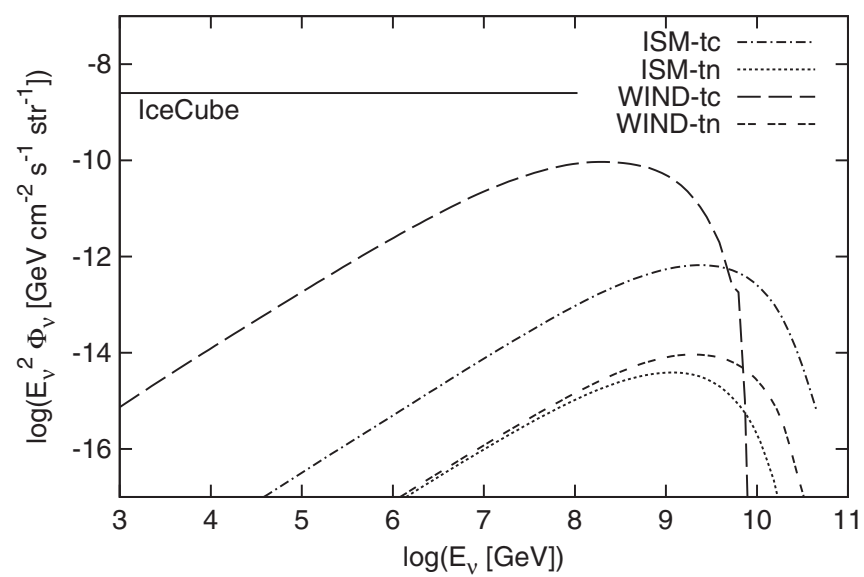

FIG. 7. The muon-neutrino $\left(\nu_{\mu}+\bar{\nu}_{\mu}\right)$ background from early afterglows of GRBs, based on the original reverse-forward shock model which typically predicts infrared/optical flashes. The normalization of the proton flux is given by $\epsilon_{\mathrm{acc}}=1 / 4$ and neutrino oscillation is taken into account.

electromagnetic observations. Such a correlation is important to detect neutrino signals from GRBs. Otherwise, neutrino signals may be buried below the atmospheric neutrino background and cosmogenic neutrino background in the high energies.

We calculate the neutrino background from GRBs for our specific parameter sets.

In Fig. 7, we show the neutrino background for the parameter sets based on the original RS model which was developed in the pre-Swift era. Even in the model WIND-tc, expected muon numbers by IceCube are $N_{\mu} \sim$ $(0.05-0.1)$ events/yr. It is very difficult to see signals for the other models in Fig. 7. Even worse, if a RS is not common, neutrino signals are also even less expected.

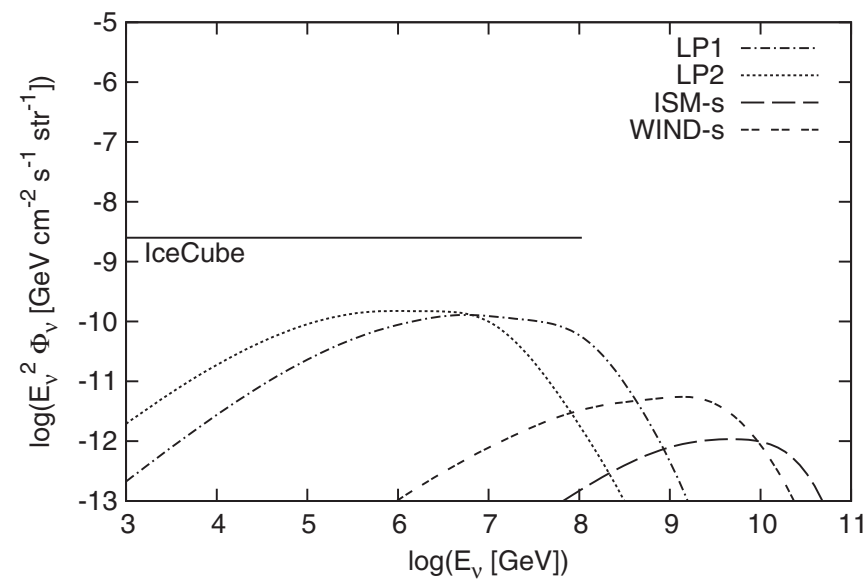

FIG. 8. The same as Fig. 7. But predictions are based on the late prompt emission model and reverse-forward shock model with the higher $\epsilon_{B}$ and smaller $f_{e}$, motivated by Swift observations. The normalization of the proton flux is given by $\xi_{\text {acc }}=10$ for the late prompt emission model, while by $\epsilon_{\mathrm{acc}}=1 / 4$ for the reverse-forward shock model.
PHYSICAL REVIEW D 76, 123001 (2007)

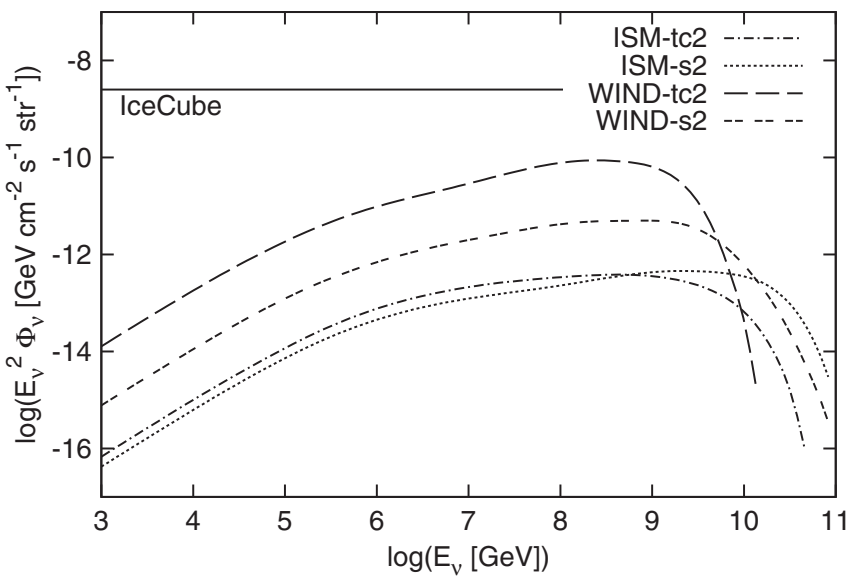

FIG. 9. The same as Figs. 7. But the RS model where the possible overlapping of the prompt emission is taken into account. The normalization of the proton flux is given by $\epsilon_{\mathrm{acc}}=$ $1 / 4$.

In Fig. 8, we show the neutrino background based on the late prompt emission model and RS model where the higher $\epsilon_{B}$ and smaller $f_{e}$ are assumed. Both are developed for explanation of the shallow decay emission. In the late prompt emission model, we can expect that the optical depth for photomeson production is high enough. In such cases, although UHECR production is difficult,

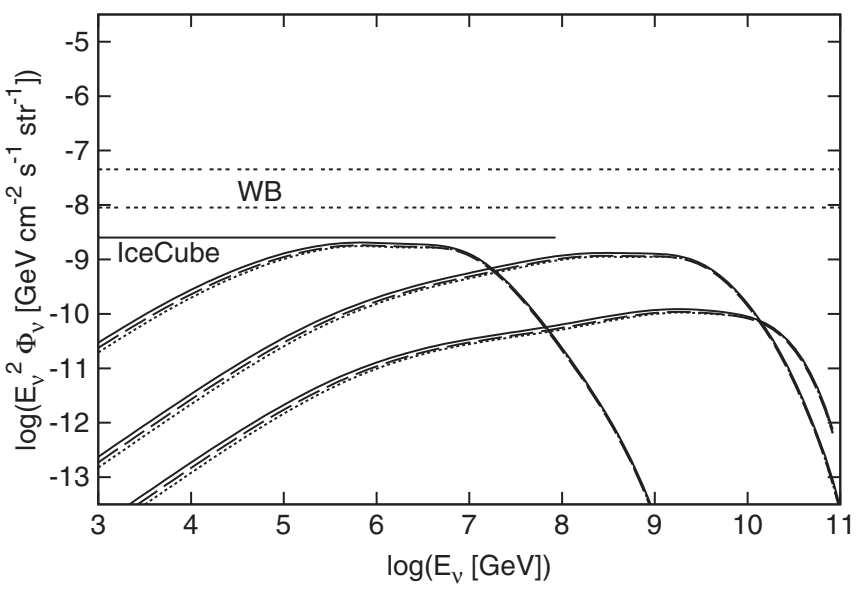

FIG. 10. The optimistic predictions of the neutrino $\left(\nu_{e}+\bar{\nu}_{e}+\right.$ $\left.\nu_{\mu}+\bar{\nu}_{\mu}+\nu_{\tau}+\bar{\nu}_{\tau}\right)$ background from early afterglows of GRBs in the Swift era. The upper three curves are for the model LP2, middle three curves for the model WIND-s2, and lower three curves for the model ISM-s2. The dependence of the neutrino backgrounds on GRB rate models is also shown. Solid curves are for the GRB3 model, dashed curves for the GRB2 model, and dotted curves for the GRB1 model (see Appendix B). In the late prompt emission model LP2, the normalization of the proton flux is given by $\xi_{\text {acc }}=50$. In the RS models ISM-s2 and WIND-s2, the possible overlapping effect due to the prompt emission is taken into account, and the proton flux is normalized to $E_{p}^{2} d \dot{N}_{p} / d E_{p}(z=0)=0.5 \times$ $10^{44} \mathrm{ergs} \mathrm{Mpc}^{-3} \mathrm{yr}^{-1}$ which corresponds to the observed UHECR flux. 
GRBs are efficient neutrino emitters. We obtain $N_{\mu} \sim$ $(0.5-1)$ events/yr for the model LP1 and $N_{\mu} \sim$ (1-3) events/yr for the model LP2, respectively. Although such signals can marginally be detected by IceCube, the significant detection requires neutrino detectors larger than IceCube. For the RS model, we have $N_{\mu} \sim$ (0.002-0.005) events/yr for the model WIND-s.

In Fig. 9, we show the neutrino background in the RS models where the possible overlapping of the prompt emission is taken into account. The effect of overlapping enhances neutrino fluxes greatly below $\sim 100 \mathrm{PeV}$.

In Fig. 10, we adopt the different normalization of the proton flux from Figs. 7-9. For the RS models, it comes from the hypothesis that the observed UHECRs come from the RS. Note that large baryon load is assumed for all the curves in Fig. 10. In addition, we have also assumed that all the GRBs are in the thick ejecta regime for the RS models. Although such assumptions may be optimistic, they can

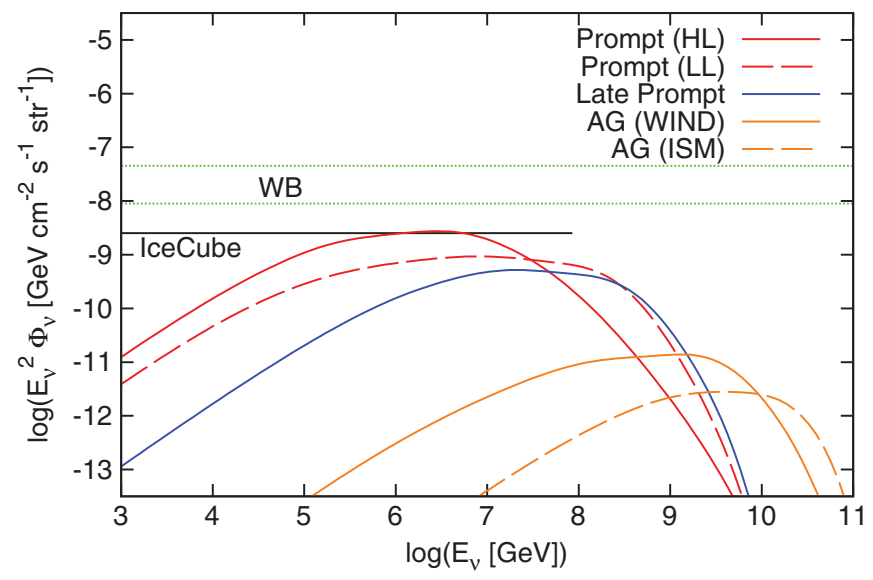

FIG. 11 (color). The fiducial predictions of the neutrino $\left(\nu_{e}+\right.$ $\bar{\nu}_{e}+\nu_{\mu}+\bar{\nu}_{\mu}+\nu_{\tau}+\bar{\nu}_{\tau}$ ) background from GRBs in the Swift era. Prompt (HL): prompt neutrino bursts from cosmological (high luminosity) GRBs; $E_{\mathrm{GRB}}=1.24 \times 10^{51} \mathrm{ergs}, \quad E_{\gamma, \mathrm{sh}}^{\text {iso }}=$ $10^{51} \mathrm{ergs}, r=10^{13-14.5} \mathrm{~cm}$, and $\Gamma_{0}=10^{2.5}, \xi_{B}=1$, and $\xi_{\text {acc }}=$ $10[1,80]$. Prompt (LL): prompt neutrino bursts from low luminosity GRBs; the local observed rate $\rho_{\mathrm{LL}}(z=0)=$ $500 \mathrm{Gpc}^{-3} \mathrm{yr}^{-1}, E_{\gamma}^{\text {iso }} \simeq 10^{50} \mathrm{ergs}, r=10^{15} \mathrm{~cm}, \Gamma_{0}=10, \xi_{B}=$ 1 , and $\xi_{\text {acc }}=10$ [54]. Late Prompt: flaring neutrino flashes and/ or neutrino early afterglows under the late prompt emission model; $\quad E_{\mathrm{LP}}=0.1 E_{\mathrm{GRB}}, \quad L_{b}=10^{48} \mathrm{ergs} \mathrm{s}^{-1}, \quad r=10^{15.3} \mathrm{~cm}$, $\Gamma_{0}=15, \xi_{B}=1$, and $\xi_{\text {acc }}=10$ (the model LP0) [53]. AG (WIND): neutrino early afterglows under the reverse-forward shock model with the windlike CBM; the model WIND-s is assumed (see the text). AG (ISM): neutrino early afterglows under the reverse-forward shock model with the ISM; the model ISM-s is assumed (see text). WB: Waxman-Bahcall bounds [77]. $\xi_{B}$ and $\xi_{\text {acc }}$ are defined as $\xi_{B} \equiv U_{B} / U_{\gamma}$ and $\xi_{\text {acc }} \equiv U_{p} / U_{\gamma}$, respectively. For the fast cooling case and the proton acceleration efficiency $\zeta_{p} \sim 1$, we have $\xi_{B} \sim\left(\epsilon_{B} / \epsilon_{e}\right)$ and $\xi_{\text {acc }} \sim 1 / \epsilon_{e}$, where $\epsilon_{e}$ is the fraction of the internal energy density carried by electrons. In all the scenarios, the GRB3 model is used (see Appendix B). lead to detectable neutrino signals from early afterglows by future observations. The dependence of the neutrino backgrounds on GRB rate models is also shown. We can see that the resulting neutrino backgrounds are not sensitive to adopted GRB rate models GRB1-GRB4 (where curves for the model GRB4 are not shown, but they are also similar to the other curves).

In Figs. 11 and 12, we show various predictions on the neutrino background from GRBs, motivated by observations in the Swift era. Figure 11 represents our fiducial predictions. For cosmological (high luminosity (HL)) GRBs, we can expect $N_{\mu} \sim(6-11)$ events/yr, which is consistent with previous works [2,5]. For low luminosity (LL) GRBs [54,79], we can expect $N_{\mu} \sim(2-5)$ events/yr, although neutrino signals coincident with LL GRBs are usually not expected by Swift and GLAST. For flares [53] and early afterglows under the late prompt emission model (the model LP0), we can expect $N_{\mu} \sim(0.4-1)$ events/yr. In the very high energy region of the neutrino background, neutrinos from LL GRBs, flares, and early afterglows will be dominant to those from HL GRBs. For AG (WIND) (the model WIND-s) and AG (ISM) (the model ISM-s), we expect $N_{\mu} \sim(0.002-0.005)$ events $/ \mathrm{yr}$ and $N_{\mu} \sim$ (0.0002-0.0004) events/yr, respectively.

Figure 12 expresses the optimistic predictions. For HL GRBs, we show the neutrino background for $\xi_{\text {acc }}=50$. The optimistic model for $\xi_{\text {acc }}=100$ is being constrained by present and future-coming neutrino observations $[80,81]$. Note that the neutrino flux is allowed to exceed the WB bound in principle if the collision radii are small enough, but the neutrino flux for Prompt (HL) would typically not exceed the WB bound $[1,77,78]$. For Prompt (LL), we normalize the proton flux to

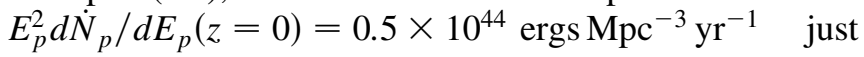

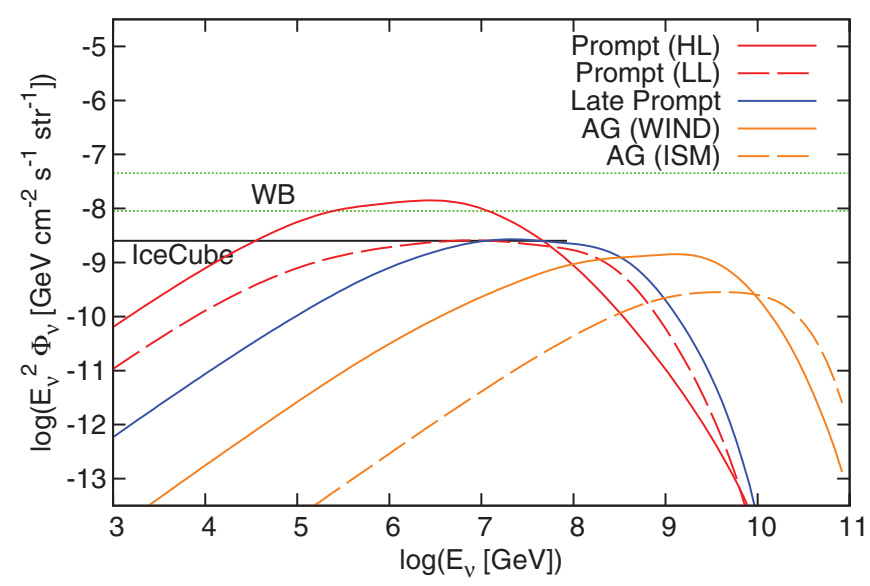

FIG. 12 (color). The same as Fig. 11, but the optimistic predictions are shown. $\xi_{\text {acc }}=50$ is used for Prompt (HL) and Late Prompt. For Prompt (LL), AG (WIND), and AG (ISM), the proton flux is normalized to $E_{p}^{2} d \dot{N}_{p} / d E_{p}(z=0)=0.5 \times$ $10^{44} \mathrm{ergs} \mathrm{Mpc}^{-3} \mathrm{yr}^{-1}$ which corresponds to the observed UHECR flux. 
for comparison. Although the shown parameter set allows protons to be accelerated up to the ultra high energy $E_{p} \sim$ $10^{20} \mathrm{eV}$, the explanation of the observed UHECRs might be difficult if UHECRs are all protons [54]. (But note that heavier nuclei could be accelerated up to the highest energies.) If accelerated protons cannot achieve the ultra high energies (below $\sim 10^{19} \mathrm{eV}$ ), the neutrino flux does not have to satisfy the WB bound in principle and could exceed the flux shown in Fig. 12 if the larger baryon load or higher rate is possible. For Late Prompt, UHECR production is typically impossible, and we just adopt $\xi_{\text {acc }}=50$. For the RS models, we normalize the proton flux to the observed UHECR flux. We have $N_{\mu} \sim(0.2-0.5)$ events $/$ yr for AG (WIND) and $N_{\mu} \sim(0.02-0.05)$ events/yr for AG (ISM).

\section{SUMMARY AND DISCUSSION}

In this paper, we have studied the neutrino emission from early afterglows of GRBs under the assumption that baryon acceleration occurs at the shocks such as the RS and late internal shocks. With acceptable parameters, we have shown that neutrino signals from early afterglows could marginally be detected by IceCube under the late prompt emission model, while they are not expected under the RS model. Hence, the neutrino detection from early afterglows will be likely to suggest that neutrinos come from the late prompt emission (including flares). Future neutrino telescopes larger than IceCube are beneficial, although the simultaneous electromagnetic multiwavelength observations are indispensable.

Our conclusions are summarized below.

(1) We have revisited the neutrino emission from the RS. We have also taken into account the cross section of photomeson production quantitatively without using the $\Delta$-resonance approximation. The effect of pion multiplicity and proton inelasticity is not important in the RS model and the $\Delta$-resonance approximation is a good approximation. The neutrino flux is suppressed in the highest energies because of the finite proton's maximum energy and cooling of secondary particles. In the original RS model, it is very difficult to detect neutrino signals for our fiducial parameter sets, even when all the GRBs accompany the RS. In addition, recent observations imply the lack of infrared/optical flashes. If it is attributed to the intrinsically weak RS, we can expect neutrino production in the RS region only for a fraction of GRBs with the sufficiently strong RS.

(2) One of the suppression mechanisms for infrared/ optical flashes is the overlapping of the prompt emission. In this scenario, the RS occurs, but cooling of electrons suppresses the emission in the infrared/optical bands. We have also taken into account this effect, which can dramatically enhance neutrino fluxes. In the case of the thick ejecta colliding into the ISM, enhancement of the flux by about 2 or 3 orders of magnitude is expected, while by 1 order of magnitude in the case of the thick ejecta colliding into the windlike CBM. Expected muon events can increase by a factor. Although this component may be hidden by prompt neutrino bursts, such a contribution will be important in the sense that we do not have to assume the proton acceleration in the region where the prompt emission occurs.

(3) Recently, it is suggested that the shallow decay emission in early afterglows may be explained by the modified RS model. As such an example, we have estimated the neutrino flux under the RS model with $f_{e}<1$. If this picture is true, we expect that the $\mathrm{RS}$ is common for all the GRBs. Therefore, we can cumulate the neutrino flux from each burst and obtain the neutrino background. We expect muon events by IceCube, $N_{\mu} \sim(0.002-0.005)$ events/yr in the model WIND-s with the moderate baryon load. The hypothesis that the observed UHECRs come from the RS leads to the higher neutrino flux, but it requires the large baryon load.

(4) One of the recently suggested models is the late prompt emission model. The physical conditions are similar to those in flares. With acceptable parameters, neutrino production can be efficient because the emission occurs at smaller radii in this model. We can have $f_{p \gamma} \gtrsim 1$, which could allow us to estimate the proton flux from the neutrino flux. In this model, we could expect detectable muon events by IceCube, although the larger telescopes would be desirable for sufficiently significant detections. If we see such signals, neutrinos will provide us with useful information on baryon acceleration in GRBs and one of the clues to the model of early afterglows.

(5) From Figs. 11 and 12, we have seen that the contribution from Prompt (HL) to the neutrino background may be the most important below $\sim 10 \mathrm{PeV}$, while the neutrino background from early afterglows can be more important in the very high energies $\gtrsim 10 \mathrm{PeV}$. Neutrino signals from early afterglows are expected to be coincident with the early afterglow emission and give us additional chances to detect high energy neutrinos. However, note that the photomeson production efficiency $f_{p \gamma}$ is sensitive to collision radii $r$ [1]. For Prompt (HL) with $\Gamma_{0} \geq 100$, we have typically $\min \left[f_{p \gamma}, 1\right] \sim$ $(0.1-1)$, and the neutrino background can be smaller than that shown in Figs. 11 or 12 where we have $f_{p \gamma} \sim 0.5$ effectively (see, e.g., the curves for the parameter set B used in Murase and Nagataki [1]). On the other hand, we obtain typically $f_{p \gamma} \gtrsim 1$ for small $\Gamma_{0} \sim 10$, which is expected in the case of the late prompt emission model with $E_{\mathrm{LP}} \sim 0.1 E_{\mathrm{GRB}}$. Therefore, the neutrino background from early 
afterglows in the late prompt emission model can be comparable to that from the prompt emission. Roughly speaking, the three possibilities (Prompt (HL), Prompt (LL), and Late Prompt) can give comparable contributions to the diffuse neutrino background.

It is important to see neutrinos associated with gamma rays. For HL GRBs, flares, and early afterglows, we can expect coincidence, while not for LL GRBs except for very nearby events. For the short-lived RS models where the overlapping of the prompt emission occurs, it will be difficult to distinguish between RS neutrinos and prompt neutrinos. This is because the short-lived RS emission for the thick ejecta case lasts for the duration of bursts $\sim T$. If the long-lived RS emission occurs, the long-term neutrino emission will be also expected. However, it may be contaminated by neutrino flashes from flares or the long-term neutrino emission from the FS. The latter could occur if protons are accelerated up to very high energies by other mechanisms such as the second-order Fermi acceleration [29-31].

Our predictions are for specific parameter sets. Of course, parameters such as $E^{b}, E_{\gamma}^{\text {iso }}$ have dispersion. A possible wide range variability may also suggest dispersion of $E_{\gamma, \mathrm{sh}}^{\text {iso }}$. Some of the parameters might be related like the $E^{b}-E_{\gamma}^{\text {iso }}$ relation for the prompt emission. More comprehensive studies are needed in the future, and more and more observations would enable us to take into account the distribution of parameters.

So far, the amount of protons has not been well constrained from observations. In Fig. 11, we have shown the relatively moderate cases, while the optimistic cases are shown in Fig. 12. Because of the lack of knowledge on the collisionless shocks and particle acceleration, we cannot say whether such optimistic choices are possible or not. But note that too large values might be implausible from recent observations [82]. Unless there is significant missing energy (which might exist, for example, if accelerated protons carry away significant energy), high radiative efficiency of GRBs implies $\epsilon_{e} \sim(0.1-1)$, which leads to $\xi_{\text {acc }} \lesssim 10$. On the other hand, the UHECR hypothesis and recently estimated local GRB rate require the large baryon load for both of the prompt and RS scenarios. We have assumed that nonthermal protons are contained, which is expected from the internal or external shock model. However, this might not be true if the outflow is Poynting dominated. The prompt or RS emission may come from magnetic dissipation processes such as reconnection, where many baryons do not have to be contained. Future neutrino observations are fruitful because they could provide us with not only information on the physics of GRB outflows but also one of the clues to unknown baryon load and acceleration in GRBs.

In this paper, we have focused on high energy neutrinos from GRBs. Generally, the high gamma-ray emission from proton-induced components such as muon synchrotron and secondary pair synchrotron should accompany neutrinos. Such high energy gamma rays can appear after complicated pair-photon cascades. Theoretically, efficient proton acceleration might induce distinctive $\mathrm{GeV}-\mathrm{TeV}$ components and such possibilities are studied by several authors [83-87]. If the overlapping of the prompt emission is common, we would expect $\mathrm{GeV}-\mathrm{TeV}$ flashes from the RS via the electron inverse-Compton scattering process [50]. In addition, the high energy emission via this process is predicted under the several early afterglow models motivated by Swift observations [88,89]. Future-coming GLAST observations [90] would also be useful for testing the models.

\section{ACKNOWLEDGMENTS}

K. M. thanks the referees, S. Nagataki, S. Yoshida, K. Ioka, K. Asano, and K. Toma for very profitable comments. The work of K. M. is supported by a Grant-in-Aid for JSPS.

\section{APPENDIX A: METHOD OF CALCULATION}

We briefly describe our method of calculation. The method is essentially the same as that used in Murase and Nagataki $[1,53]$.

The most promising acceleration process of radiating particles is the Fermi acceleration mechanism. We expect that electrons and protons are accelerated in GRBs by the Fermi acceleration mechanism. The first-order Fermi acceleration occurs by diffusive scattering of particles across strong shocks. For nonrelativistic shock acceleration, the acceleration time scale is

$$
t_{\mathrm{acc}} \approx \frac{3 r_{\mathrm{c}}}{r_{\mathrm{c}}-1} \frac{1}{U_{1}^{2}}\left(\kappa_{\mathrm{u}}+r_{\mathrm{c}} \kappa_{\mathrm{d}}\right),
$$

where $r_{\mathrm{c}}$ is the compression ratio, $U_{1}$ is the velocity of the upstream fluid, and $\kappa_{u, d}$ is the diffusion coefficients. In the ultrarelativistic shock limit, with the assumption on the sufficient amplification of the downstream magnetic field, we can obtain the acceleration time as [75],

$$
t_{\mathrm{acc}} \sim \frac{\varepsilon_{p}}{\sqrt{2} e B \Gamma_{\mathrm{rel}} c} \max \left[1,\left(\frac{\sqrt{2} \Gamma_{\mathrm{rel}} l_{\mathrm{coh}}}{r_{L}}\right)^{-1}\right],
$$

where $\Gamma_{\text {rel }}$ is the relative Lorentz factor between fluids, $l_{\text {coh }}$ is the coherent length of the upstream magnetic field, and $r_{L}$ is the Larmor radius. Let us assume the Bohm diffusion coefficient and sufficiently large coherent length of the upstream magnetic field. Then, we can write the acceleration time of protons as follows:

$$
t_{\mathrm{acc}} \equiv \eta \frac{r_{L}}{c}=\eta \frac{\varepsilon_{p}}{e B c} .
$$

For the case of mildly relativistic $\left(\Gamma_{\text {rel }} \sim\right.$ a few $)$ shocks, $\eta \sim 1$ may be possible. The acceleration time scale for the second-order Fermi acceleration is given by 


$$
t_{\mathrm{acc}} \sim\left(\frac{r_{L}}{c \beta_{A}^{2}}\right)\left(\frac{B}{\delta B}\right)^{2},
$$

where $\beta_{A} \equiv v_{A} / c, v_{A}$ is Alfvén velocity, and $\delta B$ is the strength of the turbulence in the magnetic field [73,91]. The limit $\beta_{A}=1$ and $\delta B=B$ which could be expected in the downstream of relativistic shocks also leads to the similar expression to Eq. (A3).

Proton's maximal energy is also constrained by various cooling processes. In this paper, we treat proton synchrotron cooling, inverse-Compton (IC) cooling, adiabatic cooling, and photohadronic cooling. First, the synchrotron loss time scale for relativistic protons is

$$
t_{\mathrm{syn}}=\frac{3 m_{p}^{4} c^{3}}{4 \sigma_{T} m_{e}^{2}} \frac{1}{\varepsilon_{p}} \frac{1}{U_{B}} .
$$

Second, the IC cooling time scale is given by [92]

$$
t_{\mathrm{IC}}^{-1}=\frac{c}{2 \gamma_{p}^{2}}\left(\frac{m_{e}^{2}}{m_{p}^{2}}\right) \pi r_{0}^{2} m_{p}^{2} c^{4} \int_{0}^{\infty} d \varepsilon \varepsilon^{-2} \frac{d n}{d \varepsilon} \frac{F\left(\varepsilon, \gamma_{p}\right)}{\beta_{p}\left(\gamma_{p}-1\right)},
$$

where

$$
\begin{aligned}
F(\varepsilon, \gamma) \equiv & \gamma\left[f_{1}\left(z_{a}\right)-f_{1}\left(z_{b}\right)\right]-\left(\varepsilon / m_{p} c^{2}\right) \\
& \times\left[f_{2}\left(z_{a}\right)-f_{2}\left(z_{b}\right)\right], \\
z_{a} \equiv & \frac{\varepsilon}{m_{p} c^{2}}\left(\gamma+\sqrt{\gamma^{2}-1}\right), \\
z_{b} \equiv & \frac{\varepsilon}{m_{p} c^{2}\left(\gamma+\sqrt{\gamma^{2}-1}\right)}, \\
f_{1}(z) \equiv & (z+6+3 / z) \ln (1+2 z) \\
& -\left(22 z^{3} / 3+24 z^{2}+18 z+4\right) \times(1+2 z)^{-2} \\
& -2+2 \mathrm{Li}_{2}(-2 z), \\
f_{2}(z) \equiv & \left(z+31 / 6+5 / z+3 / 2 z^{2}\right) \ln (1+2 z) \\
& -\left(22 z^{3} / 3+28 z^{2}+103 z+17+3 / z\right) \\
& \times(1+2 z)^{-2}-2+2 \operatorname{Li}_{2}(-2 z), \\
\operatorname{Li}_{2}(z)= & -\int_{0}^{z} d z^{\prime} \frac{\ln \left(1-z^{\prime}\right)}{z^{\prime}} \quad(\text { for complex } z), \\
\operatorname{Li}_{2}(z)= & \sum_{n=1}^{\infty} \frac{z^{n}}{n^{2}} \quad(\text { for }|z|<1) .
\end{aligned}
$$

Third, the photohadronic cooling time scale is

$$
t_{p \gamma}^{-1}=\frac{c}{2 \gamma_{p}^{2}} \int_{\bar{\varepsilon}_{\mathrm{th}}}^{\infty} d \bar{\varepsilon} \sigma_{p \gamma}(\bar{\varepsilon}) \kappa_{p}(\bar{\varepsilon}) \bar{\varepsilon} \int_{\bar{\varepsilon} / 2 \gamma_{p}}^{\infty} d \varepsilon \varepsilon^{-2} \frac{d n}{d \bar{\varepsilon}},
$$

where $\bar{\varepsilon}$ is the photon energy in the rest frame of proton, $\gamma_{p}$ is the proton's Lorentz factor, $\kappa_{p}$ is the proton-inelasticity, and $\bar{\varepsilon}_{\mathrm{th}}$ is the threshold photon energy for the photohadronic process in the rest frame of the incident proton. In sufficiently high energies, the photomeson cooling process is very important, where the threshold energy is $\bar{\varepsilon}_{\text {th }} \approx$
$145 \mathrm{MeV}$. We calculate Eq. (A7) by using Geant4 whose total cross section is in fairly good agreement with experimental data [55], and the calculated mean free path is also in good agreement with that obtained by the other code $[93,94]$. Fourth, we take into account the adiabatic cooling process, which has a time scale $t_{\mathrm{ad}}$ independent of the proton energy. In fact, direct ejection of protons from the emission region may depend on a proton energy if diffusive losses are relevant. For simplicity, we neglect such diffusive losses and assume that protons are confined over the time scale set by adiabatic expansion. When the fluid is relativistic, we have

$$
t_{\mathrm{ad}}^{-1}=\frac{1}{3}(\nabla \cdot \boldsymbol{V}) \sim t_{\mathrm{dyn}}^{-1}
$$

where $\boldsymbol{V}$ is the fluid velocity. From above time scales, the total proton loss time scale is expressed as $t_{p}^{-1} \equiv t_{p \gamma}^{-1}+$ $t_{\mathrm{syn}}^{-1}+t_{\mathrm{IC}}^{-1}+t_{\mathrm{ad}}^{-1}$. The proton's maximum energy can be determined by $t_{\text {acc }}<t_{p}$.

Accelerated protons interact with target photons via photomeson production. Pion spectra can be obtained by executing Geant 4 , which are written as

$$
\begin{aligned}
\frac{d n_{\pi}}{d \varepsilon_{\pi} d t}= & \int_{\varepsilon_{p}^{\min }}^{\varepsilon_{\max }^{\max }} d \varepsilon_{p} \frac{d n_{p}}{d \varepsilon_{p}} \int_{\varepsilon^{\min }}^{\varepsilon^{\max }} d \varepsilon \frac{d n}{d \varepsilon} \int \frac{d \Omega}{4 \pi} \\
& \times \frac{d \sigma_{p \gamma}\left(\varepsilon, \Omega, \varepsilon_{p}\right) \xi_{\pi}}{d \varepsilon_{\pi}} c,
\end{aligned}
$$

where $d n_{p} / d \varepsilon_{p}$ and $d n / d \varepsilon$ are proton and photon distribution in the comoving frame, $\xi_{\pi}$ is the pion-multiplicity. Owing to Geant 4 , we can include the effects of proton inelasticity and pion multiplicity. Although the treatments of photomeson production are greatly improved by using Geant4, compared to the $\Delta$-resonance approximation which is often used by other authors, Geant 4 has some problems in parametrization [1]. Therefore, we adopt the more improved approximate treatment [53,54]. Below $3 \mathrm{GeV}$, we use the experimental data [56,57] (in the previous paper [1], we approximated $\pi^{+}: \pi^{0}=1: 1$ for singlepion production, and $\pi^{+} \pi^{-}: \pi^{+} \pi^{0}=7: 4$ for double-pion production [57,73]. Both approximations do not change our conclusions). Above $3 \mathrm{GeV}$, we use the Geant4 approximation. Such an approximation is sufficient for astrophysical applications to the calculation of GRB neutrinos, and our results for neutrino spectra are quantitatively improved compared to most of the previous works, where the $\Delta$-resonance approximation is used.

We can obtain neutrino spectra from the well-known decay kinematics. Neutrinos are produced by the decay of $\pi^{ \pm} \rightarrow \mu^{ \pm}+\nu_{\mu}\left(\bar{\nu}_{\mu}\right) \rightarrow e^{ \pm}+\nu_{e}\left(\bar{\nu}_{e}\right)+\nu_{\mu}+\bar{\nu}_{\mu}$. The lifetimes of pions and muons are $t_{\pi}=\gamma_{\pi} \tau_{\pi}$ and $t_{\mu}=$ $\gamma_{\mu} \tau_{\mu}$, respectively. Here, $\tau_{\pi}=2.6033 \times 10^{-8} \mathrm{~s}$ and $\tau_{\mu}=2.1970 \times 10^{-6} \mathrm{~s}$ are the mean lifetimes of each particle. When pions decay with the spectrum $d n_{\pi} / d \varepsilon_{\pi}$, by $\pi^{ \pm} \rightarrow \mu^{ \pm}+\nu_{\mu}\left(\bar{\nu}_{\mu}\right)$, the spectrum of neutrinos is given by [95] 


$$
\frac{d n_{\nu}}{d \varepsilon_{\nu}}=\frac{m_{\pi} c}{2 \varepsilon_{\nu}^{*}} \int_{\varepsilon_{\pi}^{\min }}^{\infty} d \varepsilon_{\pi} \frac{1}{p_{\pi}} \frac{d n_{\pi}}{d \varepsilon_{\pi}},
$$

where $\varepsilon_{\nu}^{*}=\frac{\left(m_{\pi}^{2}-m_{\mu}^{2}\right) c^{2}}{2 m_{\pi}}, \varepsilon_{\pi}^{\min }=\frac{\left(\varepsilon_{\nu}^{*} / \varepsilon_{\nu}+\varepsilon_{\nu} / \varepsilon_{\nu}^{*}\right) m_{\pi} c^{2}}{2}$. Similarly, we can get the muon spectrum from the pion spectrum. Muon decay is the three-body-decay process, which is slightly more complicated than the case of two-body decay. Given the spectrum of muon, it can be calculated by the following equation [95],

$$
\begin{aligned}
\frac{d n_{\nu}}{d \varepsilon_{\nu}}= & \int_{\varepsilon_{\mu}^{\min }}^{\infty} d \varepsilon_{\mu} \frac{1}{c p_{\mu}} \frac{d n_{\mu}}{d \varepsilon_{\mu}} \int_{\varepsilon_{\nu 1}^{*}}^{\varepsilon_{\nu 2}^{*}} d \varepsilon_{\nu}^{*} \frac{1}{\varepsilon_{\nu}^{*}} \\
& \times\left(f_{0}\left(\varepsilon_{\nu}^{*}\right) \mp \cos \theta_{\nu}^{*} f_{1}\left(\varepsilon_{\nu}^{*}\right)\right),
\end{aligned}
$$

where $\varepsilon_{\nu 1}^{*}=\gamma_{\mu} \varepsilon_{\nu}-\left(\gamma_{\mu}^{2}-1\right)^{1 / 2} \varepsilon_{\nu}, \varepsilon_{\nu 2}^{*}=\min \left[\gamma_{\mu} \varepsilon_{\nu}+\right.$ $\left.\left(\gamma_{\mu}^{2}-1\right)^{1 / 2} \varepsilon_{\nu},\left(m_{\mu}^{2}-m_{e}^{2}\right) c^{2} / 2 m_{\mu}\right], \quad \varepsilon_{\nu}^{*}=\frac{m_{\mu}^{2}-m_{e}^{2}}{2 m_{\mu}} c^{2}$ that are defined in the muon rest frame and for muon neutrinos, $f_{0}(x)=2 x^{2}(3-2 x), f_{1}(x)=2 x^{2}(1-2 x)$, and for electron neutrinos, $f_{0}(x)=12 x^{2}(1-x), f_{1}(x)=12 x^{2}(1-$ $x$ ), where $x \equiv 2 \varepsilon_{\nu}^{*} / m_{\mu} c^{2}$ and $\theta_{\nu}^{*}$ is the angle between the muon spin and the direction of a neutrino.

Because of cooling processes of $\pi^{ \pm}$and $\mu^{ \pm}$, we have to apply Eqs. (A10) and (A11) at each time step, when we solve the following equation:

$$
\begin{gathered}
\frac{\partial}{\partial t}\left(\frac{d n_{\pi, \mu}}{d \varepsilon_{\pi, \mu}}\left(\varepsilon_{\pi, \mu}, t\right)\right)+\frac{\partial}{\partial \varepsilon_{\pi, \mu}}\left(\dot{\varepsilon}_{\pi, \mu} \frac{d n_{\pi, \mu}}{d \varepsilon_{\pi, \mu}}\left(\varepsilon_{\pi, \mu}, t\right)\right) \\
=-\frac{1}{t_{\pi, \mu}} \frac{d n_{\pi, \mu}}{d \varepsilon_{\pi, \mu}}\left(\varepsilon_{\pi, \mu}, t\right)+Q_{\pi, \mu}\left(\varepsilon_{\pi, \mu}, t\right),
\end{gathered}
$$

where $Q_{\pi, \mu}$ represents the source term of pions and muons due to photomeson production and decay of pions, respectively. The synchrotron cooling time scale is given by replacing proton mass with pion or muon mass in Eq. (A6). The adiabatic cooling time scale is still comparable to the dynamical time scale. We also treat the IC process including the Klein-Nishina effect for pions and muons. We neglect the $\pi \gamma$ process (including the photomeson production and photopair production), because other cooling processes are usually more important in our interested cases. Throughout the calculations, we also neglect neutrinos from neutron decay $n \rightarrow p+e^{-}+\bar{\nu}_{e}$, whose time scale is usually much larger than the dynamical time scale $t_{\mathrm{dyn}}$ for the short-lived emission (but note that these components will also contribute to the diffuse neutrino background).

\section{APPENDIX B: GRB NEUTRINO BACKGROUND}

In order to get the differential number flux of background neutrinos, first we compute the present number density of the background neutrinos per unit energy from one GRB. The contribution of neutrinos emitted in the interval of the redshift $z \sim z+d z$ is given as $d n_{\nu}^{\mathrm{ob}}\left(E_{\nu}\right)=R_{\mathrm{GRB}}(z)(1+z)^{3} \frac{d t}{d z} d z \frac{d N_{\nu}\left(E_{\nu}^{\prime}\right)}{d E_{\nu}^{\prime}} d E_{\nu}^{\prime}(1+z)^{-3}$,

where

$$
\frac{d t}{d z}=-\frac{1}{H_{0}(1+z)} \frac{1}{\sqrt{\Omega_{\Lambda}+\bar{\Omega}_{k}(1+z)^{2}+\bar{\Omega}_{m}(1+z)^{3}}},
$$

and $E_{\nu}^{\prime}=(1+z) E_{\nu}$ is the energy of neutrinos at redshift $z$, which is now observed as $E_{\nu}$ and $d N_{\nu}\left(E_{\nu}^{\prime}\right) / d E_{\nu}^{\prime}$ is the number spectrum of neutrinos emitted by one GRB event. Hence, the GRB neutrino background can be calculated using the following equation:

$$
\begin{aligned}
\Phi_{\nu} \equiv & \frac{c d n_{\nu}^{\mathrm{ob}}}{d E_{\nu} d \Omega} \\
= & \frac{c}{4 \pi H_{0}} \int_{0}^{z_{\max }} d z R_{\mathrm{GRB}}(z) \frac{d N_{\nu}\left((1+z) E_{\nu}\right)}{d E_{\nu}^{\prime}} \\
& \times \frac{1}{\sqrt{\Omega_{\Lambda}+\Omega_{k}(1+z)^{2}+\Omega_{m}(1+z)^{3}}} .
\end{aligned}
$$

In this paper, we adopt $z_{\max }=11, \Omega_{m}=0.3, \Omega_{k}=0$, $\Omega_{\Lambda}=0.7$, and $H_{0}=71 \mathrm{~km} \mathrm{~s}^{-1} \mathrm{Mpc}^{-1}$. Here, $R_{\mathrm{GRB}}(z)$ is the beaming-corrected (overall) GRB rate. The local GRB rate has some uncertainties depending on the rate history, but our results on the neutrino background are not so changed because the main contribution to the background comes from GRBs that occur at $z \sim(1-3)$, the number of which is observationally determined. We use the following GRB rate history in units of $\mathrm{yr}^{-1} \mathrm{Gpc}^{-3}[1,96]$,

$$
\begin{aligned}
& R_{\mathrm{GRB} 1}(z)=18 \frac{46 \mathrm{e}^{3.4 z}}{\mathrm{e}^{3.8 z}+45} F\left(z, \Omega_{m}, \Omega_{\Lambda}\right), \\
& R_{\mathrm{GRB} 2}(z)=18 \frac{23 \mathrm{e}^{3.4 z}}{\mathrm{e}^{3.4 z}+22} F\left(z, \Omega_{m}, \Omega_{\Lambda}\right), \\
& R_{\mathrm{GRB} 3}(z)=23 \frac{24 \mathrm{e}^{3.05 z-0.4}}{\mathrm{e}^{2.93 z}+15} F\left(z, \Omega_{m}, \Omega_{\Lambda}\right), \\
& R_{\mathrm{GRB} 4}(z)=43 F\left(z, \Omega_{m}, \Omega_{\Lambda}\right)\left\{\begin{array}{l}
10^{0.75 z}(z<1) \\
10^{0.75}(z>1)
\end{array},\right.
\end{aligned}
$$

where $F\left(z, \Omega_{m}, \Omega_{\Lambda}\right)=\sqrt{\Omega_{\Lambda}+\Omega_{m}(1+z)^{3}} /(1+z)^{3 / 2}$. In this paper, we show results calculated under the GRB3 model in most figures, because the dependence of the resulting neutrino backgrounds on GRB rate models is not so large [1]. In the previous work [1], we evaluated the neutrino background by exploiting Eqs. (B4) with the beaming-corrected energy $E_{\mathrm{GRB}}=1.24 \times 10^{51}$ ergs. Alternatively, when we have isotropic energy, we should use the apparent GRB rate which is expressed as $\rho_{\mathrm{GRB}} \equiv$ $f_{b} R_{\mathrm{GRB}}$. In this paper, we adopt $f_{b}=1 / 75$ as a fiducial value for evaluation of the neutrino background from the RS. 


\section{APPENDIX C: VACUUM NEUTRINO OSCILLATION}

Neutrino physics has been interesting since neutrino experiments recently discovered something new, rather than giving only more precise measurements of standard model parameters, or stronger bounds on unseen new physics. Solar and atmospheric neutrino data directly show that the flux of neutrinos with each flavor is not conserved, which suggests that neutrinos are massive then there should be a neutrino mixing matrix.

In GRBs, neutrinos are produced via photomeson production, so that we can expect $\nu_{e}: \nu_{\mu}: \nu_{\tau} \approx 1: 2: 0$ approximately. As a result of neutrino oscillation, we can obtain $\nu_{e}: \nu_{\mu}: \nu_{\tau} \approx 1: 1: 1$. So there may be a possibility that tau neutrinos are detected through double bang events [97]. However, we should note that the magnetic field is strong in GRBs, so that contributions from muons that are more

[1] K. Murase and S. Nagataki, Phys. Rev. D 73, 063002 (2006).

[2] E. Waxman and J. Bahcall, Phys. Rev. Lett. 78, 2292 (1997).

[3] K. Asano, Astrophys. J. 623, 967 (2005).

[4] C. D. Dermer and A. Atoyan, Phys. Rev. Lett. 91, 071102 (2003).

[5] D. Guetta, D. W. Hooper, J. Alvarez-Muñiz, F. Halzen, and E. Reuveni, Astropart. Phys. 20, 429 (2004).

[6] T. Piran, Phys. Rep. 314, 575 (1999).

[7] T. Piran, Rev. Mod. Phys. 76, 1143 (2005).

[8] B. Zhang and P. Mészáros, Int. J. Mod. Phys. A 19, 2385 (2004).

[9] S. R. Kulkarni et al., Nature (London) 398, 389 (1999).

[10] C. Akerlof et al., Nature (London) 398, 400 (1999).

[11] R. Sari and T. Piran, Astrophys. J. 517, L109 (1999).

[12] P. Mészŕos and M. J. Rees, Mon. Not. R. Astron. Soc. 306, L39 (1999).

[13] A. Panaitescu and P. Kumar, Astrophys. J. 560, L49 (2001).

[14] A. M. Soderberg and E. Ramirez-Ruiz, Mon. Not. R. Astron. Soc. 330, L24 (2002).

[15] Y.Z. Fan et al., Chin. J. Astron. Astrophys. 2, 449 (2002).

[16] B. Zhang, S. Kobayashi, and P. Mészáros, Astrophys. J. 595, 950 (2003).

[17] A. Panaitescu and P. Kumar, Mon. Not. R. Astron. Soc. 353, 511 (2004).

[18] E. Nakar and T. Piran, Astrophys. J. 619, L147 (2005).

[19] D. Fox et al., Astrophys. J. 586, L5 (2003).

[20] D. M. Wei, Astron. Astrophys. 402, L19 (2003).

[21] P. Kumar and A. Panaitescu, Mon. Not. R. Astron. Soc. 346, 905 (2003).

[22] D. Fox et al., Nature (London) 422, 284 (2003).

[23] S. Kobayashi and B. Zhang, Astrophys. J. 582, L75 (2003). subject to cooling due to their longer lifetime, are suppressed. Hence, we expect $\nu_{e}: \nu_{\mu}: \nu_{\tau} \approx 0: 1: 0$ in the high energy region. As a result of neutrino oscillation, we can obtain $\nu_{e}: \nu_{\mu}: \nu_{\tau} \approx 1: 1.8: 1.8$ rather than $\nu_{e}: \nu_{\mu}: \nu_{\tau} \approx 1: 1: 1$ in the high energy region [98].

In this paper, we consider vacuum neutrino oscillation in the long baseline limit. Assuming that $\theta_{23}$ is maximal $\left(\theta_{23} \approx \pi / 4\right)$ and $\theta_{13}$ is very small $\left(\theta_{13} \approx 0\right)$, which are indicated by neutrino oscillation data, we can obtain,

$$
\begin{aligned}
\Phi_{\nu_{e}} & \approx \Phi_{\nu_{e}}^{0}-\frac{1}{4} \sin ^{2} 2 \theta_{12}\left(2 \Phi_{\nu_{e}}^{0}-\Phi_{\nu_{\mu}}^{0}-\Phi_{\nu_{\tau}}^{0}\right), \quad(\mathrm{C} 1) \\
\Phi_{\nu_{\mu}} & \approx \Phi_{\nu_{\tau}} \\
& \approx \frac{1}{2}\left(\Phi_{\nu_{\mu}}^{0}+\Phi_{\nu_{\tau}}^{0}\right)+\frac{1}{8} \sin ^{2} 2 \theta_{12}\left(2 \Phi_{\nu_{e}}^{0}-\Phi_{\nu_{\mu}}^{0}-\Phi_{\nu_{\tau}}^{0}\right) .
\end{aligned}
$$

In this paper, we adopt $\theta_{12}=0.59$.
[24] C. H. Blake et al., Nature (London) 435, 181 (2005).

[25] W. T. Vestrand et al., Nature (London) 435, 178 (2005).

[26] Y.Z. Fan et al., Astrophys. J. 628, 1, L25 (2005).

[27] E. Waxman and J. Bahcall, Astrophys. J. 541, 707 (2000).

[28] Z. G. Dai and T. Lu, Astrophys. J. 551, 249 (2001).

[29] C. D. Dermer, Astrophys. J. 574, 65 (2002).

[30] C. D. Dermer, Astrophys. J. 664, 384 (2007).

[31] Z. Li, Z. G. Dai, and T. Lu, Astron. Astrophys. 396, 303 (2002).

[32] J. Ahrens et al., Astropart. Phys. 20, 507 (2004).

[33] U.F. Katz, Nucl. Instrum. Methods Phys. Res., Sect. A 567, 457 (2006).

[34] S. W. Barwick et al., Phys. Rev. Lett. 96, 171101 (2006).

[35] V. Van Elewyck et al., arXiv:astro-ph/0612731.

[36] J. Granot, Nuovo Cimento Soc. Ital. Fis. B 121, 1073 (2006).

[37] P. Mészáros, Rep. Prog. Phys. 69, 2259 (2006).

[38] B. Zhang et al., Astrophys. J. 642, 354 (2006).

[39] B. Zhang, Chin. J. Astron. Astrophys. 7, 1 (2007).

[40] P. T. O’Brien et al., Astrophys. J. 647, 1213 (2006).

[41] R. Willingale et al., Astrophys. J. 662, 1093 (2007).

[42] K. Ioka, S. Kobayashi, and B. Zhang, Astrophys. J. 631, 429 (2005).

[43] D. N. Burrows et al., Phil. Trans. R. Soc. A 365, 1213 (2007).

[44] A. D. Falcone et al., arXiv:0706.1564.

[45] G. Ghisellini et al., Astrophys. J. 658, L75 (2007).

[46] P. W. A. Roming et al., Astrophys. J. 652, 1416 (2006).

[47] E. Nakar and T. Piran, Mon. Not. R. Astron. Soc. 353, 647 (2004).

[48] E. McMahon, P. Kumar, and T. Piran, Mon. Not. R. Astron. Soc. 366, 575 (2006).

[49] B. Zhang and S. Kobayashi, Astrophys. J. 628, 315 (2005).

[50] A. M. Beloborodov, Astrophys. J. 618, L13 (2005). 
[51] F. Genet, F. Daigne, and R. Mochkovitch, Mon. Not. R. Astron. Soc. 381, 732 (2007).

[52] L. Z. Uhm and A. M. Beloborodov, Astrophys. J. 665, L93 (2007).

[53] K. Murase and S. Nagataki, Phys. Rev. Lett. 97, 051101 (2006).

[54] K. Murase, K. Ioka, S. Nagataki, and T. Nakamura, Astrophys. J. 651, L5 (2006).

[55] S. Agostinelli et al., Nucl. Instrum. Methods Phys. Res., Sect. A 506, 250 (2003); http://wwwasd.web.cern.ch/ wwwasd/geant4/geant4.html.

[56] Particle Data Group, http://pdg.lbl.gov/.

[57] S. Schadmand Eur. Phys. J. A 18, 405 (2003).

[58] R. A. Chevalier and Z. Y. Li, Astrophys. J. 536, 195 (2000).

[59] R. Sari and T. Piran, Astrophys. J. 455, L143 (1995).

[60] S. Kobayashi, Astrophys. J. 545, 807 (2000).

[61] A. Panaitescu and P. Kumar, Astrophys. J. 543, 66 (2000).

[62] R. Wijers and T. Galama, Astrophys. J. 523, 177 (1999).

[63] J. Granot, T. Piran, and R. Sari, Astrophys. J. 534, L163 (2000).

[64] D. Eichler and E. Waxman, Astrophys. J. 627, 861 (2005).

[65] M. Milosavljević and E. Nakar, Astrophys. J. 641, 978 (2006).

[66] Y.Z. Fan, B. Zhang, and D. M. Wei, Astrophys. J. 629, 334 (2005).

[67] Y.Z. Fan and D. M. Wei, Mon. Not. R. Astron. Soc. 364, L42 (2006).

[68] E. Troja et al., Astrophys. J. 665, 599 (2007).

[69] E. W. Liang, B. B. Zhang, and B. Zhang, Astrophys. J. 670, 565 (2007).

[70] D. Guetta and T. Piran, J. Cosmol. Astropart. Phys. 07 (2007) 003.

[71] A. Achterberg, Y.A. Gallant, J.G. Kirk, and A. W. Guthmann, Mon. Not. R. Astron. Soc. 328, 393 (2001).

[72] U. Keshet and E. Waxman, Phys. Rev. Lett. 94, 111102 (2005).

[73] J.P. Rachen and P. Mészáros, Phys. Rev. D 58, 123005 (1998).

[74] A. M. Hillas, Annu. Rev. Astron. Astrophys. 22, 425 (1984).
[75] Y. A. Gallant and A. Achterberg, Mon. Not. R. Astron. Soc. 305, L6 (1999).

[76] C. D. Dermer and M. Humi, Astrophys. J. 556, 479 (2001).

[77] E. Waxman and J. Bahcall, Phys. Rev. D 59, 023002 (1998).

[78] K. Mannheim, R. J. Protheroe, and J. P. Rachen, Phys. Rev. D 63, 023003 (2000).

[79] N. Gupta and B. Zhang, Astropart. Phys. 27, 386 (2007).

[80] A. Achterberg et al., Astrophys. J. 664, 397 (2007).

[81] A. Achterberg et al., arXiv:0705.1186.

[82] B. Zhang et al., Astrophys. J. 655, 989 (2007).

[83] M. Böttcher and C. D. Dermer, Astrophys. J. 499, L131 (1998).

[84] C. D. Dermer and A. Atoyan, New J. Phys. 8, 122 (2006).

[85] C. D. Dermer, E. Ramirez-Ruiz, and T. Le, arXiv:astro-ph/ 0703219.

[86] A. Peér and E. Waxman, Astrophys. J. 633, 1018 (2005).

[87] B. Zhang and P. Mészáros, Astrophys. J. 559, 110 (2001).

[88] Y.Z. Fan et al., arXiv:0704.2063.

[89] X. Y. Wang, Z. Li, and P. Mész'aros, Astrophys. J. 641, L89 (2006).

[90] J.E. McEnery, I. V. Moskalenko, and J.F. Ormes, in Cosmic Gamma-Ray Sources, edited by K. S. Cheng and G. E. Romero, Astrophysics and Space Science Library, Vol. 304 (Kluwer, Dordrecht, 2004), p. 361.

[91] R. M. Kulslud, in Particle Acceleration Mechanisms in Astrophysics, edited by J. Arons, C. Max, and C. Mckee, AIP Conf. Proc. No. 56 (AIP, New York, 1979), p. 13.

[92] F. C. Jones, Phys. Rev. 137, B1306 (1965).

[93] A. Mücke et al., Pub. Astron. Soc. Aust. 16, 160 (1999).

[94] H. Takami, K. Murase, S. Nagataki, and K. Sato, arXiv:0704.0979.

[95] R. Schlickeiser, Cosmic Ray Astrophysics, A\&A Library (Springer-Verlag, Berlin, 2003).

[96] D. Guetta, T. Piran, and E. Waxman, Astrophys. J. 619, 412 (2005).

[97] H. Athar, G. Parente, and E. Zas, Phys. Rev. D 62, 093010 (2000).

[98] T. Kashti and E. Waxman, Phys. Rev. Lett. 95, 181101 (2005). 